\title{
Variants of the human RAD52 gene confer defects in ionizing radiation resistance and homologous recombination repair in budding yeast
}

\author{
Alissa D. Clear ${ }^{1,2,3}$, Glenn M. Manthey ${ }^{1,2}$, Olivia Lewis ${ }^{4,5}$, Isabelle Y. Lopez ${ }^{4,6}$, Rossana Rico ${ }^{4,7}$, Shannon \\ Owens $^{8,9}$, M. Cristina Negritto ${ }^{10}$, Elise W. Wolf ${ }^{10,11}$, Jason $\mathrm{Xu}^{10,12}$, Nikola Kenjić ${ }^{13}$, J. Jefferson P. Perry ${ }^{13}$, \\ Aaron W. Adamson ${ }^{14}$, Susan L. Neuhausen ${ }^{14}$, Adam M. Bailis ${ }^{1,2,15, *}$ \\ ${ }^{1}$ Department of Molecular and Cellular Biology, Beckman Research Institute of City of Hope, Duarte, CA, USA. \\ 2 Irell \& Manella Graduate School of Biological Sciences, Beckman Research Institute of City of Hope, Duarte, CA, USA. \\ ${ }^{3}$ bioStrategies Group, Chicago, IL, USA. \\ ${ }^{4}$ City of Hope - Duarte High School NIH Science Education Partnership Award Program, Duarte, CA, USA. \\ 5 Barbara Bush Houston Literacy Foundation, Houston, TX, USA. \\ ${ }^{6}$ California State Polytechnic University at Pomona, Pomona, CA, USA. \\ ${ }^{7}$ Henry Samueli School of Engineering and Applied Sciences, University of California at Los Angeles, Los Angeles, CA, USA. \\ 8 Eugene and Ruth Roberts Summer Student Academy, Beckman Research Institute of City of Hope, Duarte, CA, USA. \\ ${ }^{9}$ Department of Microbiology and Molecular Genetics, College of Biological Sciences, University of California at Davis, Davis, CA, USA. \\ ${ }^{10}$ Molecular Biology Program, Pomona College, Claremont, CA, USA. \\ ${ }^{11}$ Department of Microbiology and Immunology, University of California at San Francisco, San Francisco, CA, USA. \\ 12 Perelman School of Medicine, University of Pennsylvania, Philadelphia, PA, USA. \\ ${ }_{13}$ Department of Biochemistry, University of California at Riverside, Riverside, CA, USA. \\ ${ }^{14}$ Department of Population Sciences, Beckman Research Institute of City of Hope, Duarte, CA, USA. \\ ${ }^{15}$ College of Health Professions, Thomas Jefferson University, Philadelphia, PA, USA. \\ * Corresponding Author: \\ Adam M. Bailis, Ph.D., College of Health Professions, Thomas Jefferson University, 130 S 9th St, Philadelphia, PA 19107; Tel - (215) \\ 503-4943; Fax- (215) 503-5819; E-mail: adam.bailis@jefferson.edu
}

ABSTRACT RAD52 is a structurally and functionally conserved component of the DNA double-strand break (DSB) repair apparatus from budding yeast to humans. We recently showed that expressing the human gene, HsRAD52 in rad52 mutant budding yeast cells can suppress both their ionizing radiation (IR) sensitivity and homologous recombination repair (HRR) defects. Intriguingly, we observed that HsRAD52 supports DSB repair by a mechanism of HRR that conserves genome structure and is independent of the canonical HR machinery. In this study we report that naturally occurring variants of HsRAD52, one of which suppresses the pathogenicity of BRCA2 mutations, were unable to suppress the IR sensitivity and HRR defects of rad52 mutant yeast cells, but fully suppressed a defect in DSB repair by single-strand annealing (SSA). This failure to suppress both IR sensitivity and the HRR defect correlated with an inability of HsRAD52 protein to associate with and drive an interaction between genomic sequences during DSB repair by HRR. These results suggest that HsRAD52 supports multiple, distinct DSB repair apparatuses in budding yeast cells and help further define its mechanism of action in HRR. They also imply that disruption of HsRAD52-dependent HRR in BRCA2defective human cells may contribute to protection against tumorigenesis and provide a target for killing BRCA2-defective cancers. doi: $10.15698 /$ mic2020.10.732

Received originally: 20.05.2020;

in revised form: 03.07.2020,

Accepted 14.07.2020,

Published 20.07.2020.

Keywords: HsRAD52 variants, tumorigenesis, DNA double strand breaks, homologous recombination repair, ionizing radiation, budding yeast.

\section{Abbreviations:}

$D R R$-direct repeat recombination; $D S B$ - double-strand break; HsRAD52 - human RAD52; $H R$ - homologous recombination; $H R R-H R$ repair; $I R$ - ionizing radiation; MTI - mating type interconversion; NHEJ - non-homologous end joining; SCRAD52 - yeast RAD52. 


\section{INTRODUCTION}

lonizing radiation (IR) is a ubiquitous component of our environment that arises from both natural and manmade sources. Human exposure to IR results in damage at the cellular level, with DNA damage thought to be the source of its lethal effect. IR induces a variety of physical and chemical changes to the DNA, with the double-strand break (DSB) being the most lethal [1-4]. Accordingly, biological systems have evolved multiple mechanisms for the repair of DSBs that contribute to IR resistance [5-11]. The most prominent of these mechanisms are homologous recombination repair (HRR) and non-homologous end joining (NHEJ), which are genetically and biochemically distinct. These distinctions are the basis of the different outcomes of DSB repair by HRR and NHEJ, with HRR most frequently conserving genome structure and NHEJ frequently altering it [12-14]. The balance between these conservative and non-conservative mechanisms of DSB repair can have profound effects on genome integrity and human health following exposure to IR [15].

Intriguingly, the balance between conservative HRR and non-conservative NHEJ in DSB repair favors NHEJ in human cells, perhaps reflecting the extraordinary speed and efficiency of the mechanism [16]. However, mutations in several HRR genes, including ATM, MRE11, and XRCC3 can increase sensitivity to IR and cancer susceptibility [17], indicating the relevance of DSB repair by HRR in the response of human cells to IR exposure. An interesting facet of DSB repair by HRR was revealed when attenuation of the HR factor, RAD52, in addition to canonical HRR pathway defects had profoundly synergistic effects on DSB repair by HRR, suggesting that it may support a distinct mechanism of HRR [18-21]. Independent localization of RAD52 and canonical HRR factors to IR-induced DNA damage confirmed their distinct cellular response to DSBs and the probable existence of separate repair apparatuses [22]. The synthetic lethality observed upon simultaneous attenuation of RAD52 and various canonical HRR factors, particularly BRCA1 and BRCA2 points to potential therapeutic opportunities presented by disrupting the activities of both apparatuses [18-21, 23-28], which may include sensitization of tumors to therapeutic IR.

Advancing the development of effective inhibitors of RAD52 would benefit from a more complete understanding of its function at the molecular level during DSB repair in living cells. Studying the function of human RAD52 (hereafter referred to as HsRAD52) in IR resistance and HRR in budding yeast cells has provided a compelling model as our previous investigation showed that expressing the HsRAD52 gene in rad52 mutant yeast strains suppresses both their IR sensitivity and HRR defects [29]. Further, HsRAD52-dependent HRR and association of HsRAD52 with DSBs during repair are independent of the central HR factor, Rad51 and other members of the canonical HRR apparatus of yeast, paralleling observations in mammalian cells. In the current study we have used naturally occurring variants of HSRAD52 identified in African American women with breast cancer, HsRAD52-G59R and HsRAD52-S346X to further study the control of HsRAD52-dependent HRR at the genetic and molecular levels in budding yeast. We found that neither allele suppressed the IR sensitivity or defective DSB repair by conservative HRR of rad52 mutant cells. Our recent observation that HsRAD52-S346X protects against breast cancer in carriers of pathogenic BRCA2 mutations [30] suggests the possibility that a similar loss of HsRAD52-dependent HRR in human cells may contribute to the synthetic lethality that underlies this protection. Together, these observations further demonstrate the utility of the yeast model system for studying the function of HsRAD52 at the DNA level, and highlight a potential avenue for exploring the mechanisms by which inhibitors block DSB repair in living cells.

\section{RESULTS}

Expression of the HsRAD52-G59R and HsRAD52-S346X variant alleles in budding yeast cells produce stable proteins

As HsRAD52 plays a role in DSB repair by HRR [19-21] and defects in HRR are linked to cancer susceptibility [31-34], we reasoned that variants of $H s R A D 52$ from patients with cancer might confer loss of protein function. Accordingly, we examined the effects of HsRAD52-G59R and HsRAD52S346X, two variants identified in a screen of AfricanAmerican women with breast cancer, for their effects on the function of HsRAD52 in budding yeast cells. Utilizing our previous strategy for obtaining robust expression of HsRAD52 in yeast [29], we inserted CDNAs of the HsRAD52G59R and HsRAD52-S346X alleles into the $A D H 1$ locus such that their expression was controlled by the $A D H 1$ promoter and terminator sequences. Strains expressing C-terminally

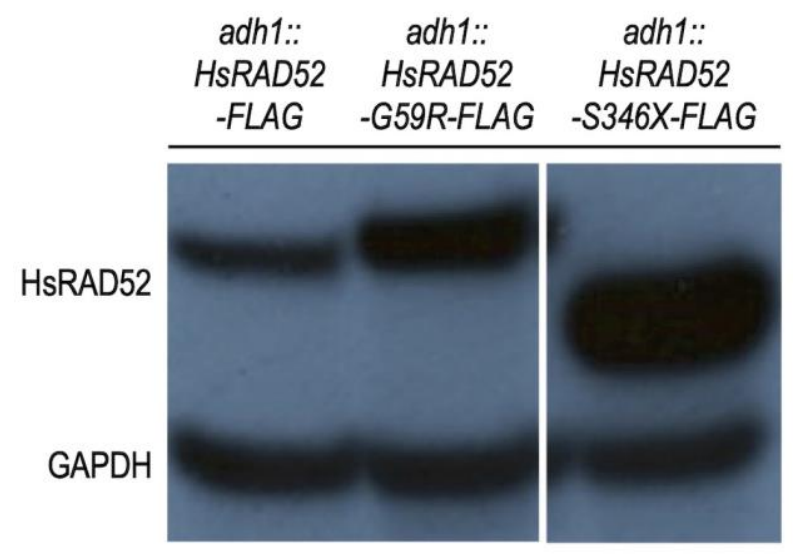

FIGURE 1: The adh1::HsRAD52-G59R-FLAG and adh1::HsRAD52S346X-FLAG alleles express stable proteins in budding yeast cells. Whole cell extracts from strains ABX3684-12B (adh1::HsRAD52-FLAG), ABX3782-2D (adh1::HsRAD52-G59RFLAG) and ABX3974-11C (adh1::HsRAD52-S346X-FLAG) were run on SDS-PAGE gels, blotted to a nylon membrane and probed with anti-FLAG and anti-GAPDH antibodies. The genotypes of the strains are denoted at the top of the figure. Bands corresponding to wild-type and mutant HsRAD52, and GAPDH are labeled on the left side of the figure. 
FLAG-tagged recombinants also were produced in order to facilitate immunological detection and selection of the proteins. As observed previously with strains expressing the adh1::HsRAD52-FLAG allele [29], Western blots of whole cell extracts from strains expressing the adh1::HsRAD52-G59R-FLAG allele produced a single, stable $49 \mathrm{kDa}$ protein, with the steady-state level of the mutant protein appearing to exceed that of wild-type (Figure 1). Strains expressing the adh1::HsRAD52-S346X-FLAG allele displayed a single $38 \mathrm{kDa}$ protein consistent with the deletion of 72 amino acids from the C-terminus of HsRAD52. The truncated mutant protein accumulated to a significantly higher level than either of the full-length proteins.

The HsRAD52-G59R and HsRAD52-S346X alleles do not suppress the IR sensitivity of rad52 mutant yeast cells In budding yeast cells Rad52 (hereafter referred to as ScRad52) is the central factor controlling HRR, playing a predominant role in maintaining IR resistance as indicated by the exquisite sensitivity to gamma and $\mathrm{X}$-ray radiation displayed by rad52 mutant strains $[9,35,36]$. Consistent with these and our own results using a gamma radiation source [29], we observed a 767-fold decrease in viability of a rad52 $\%$ homozygous mutant diploid strain after exposure to $320 \mathrm{~Gy}$ of X-ray radiation relative to a wild-type diploid (Figure 2; Table S4). Importantly, expression of adh1::HsRAD52-FLAG in a rad52\% mutant diploid suppressed its sensitivity to $320 \mathrm{~Gy}$ of $\mathrm{X}$-ray radiation by greater than 20-fold. This indicates that HsRAD52-FLAG can participate in the repair of X-ray-induced DSBs in budding yeast cells.

Having established that the adh1::HsRAD52-G59R-FLAG and adh1::HsRAD52-S346X-FLAG alleles express stable proteins at steady-state levels that are at least equal to that expressed by adh1::HsRAD52-FLAG (Figure 1), we examined their ability to suppress the IR sensitivity of the rad52\% mutant diploid. In contrast to expression of the adh1:: HsRAD52-FLAG allele, we observed that expression of the adh1::HsRAD52-G59R-FLAG and adh1::HsRAD52S346X-FLAG mutant alleles in rad52\% mutant diploid strains failed to significantly suppress IR sensitivity at any $X$-ray dose (Figure 2; Table S4; $p>0.76$ ). This indicates that the mutant HsRAD52-G59R-FLAG and HsRAD52-S346X-

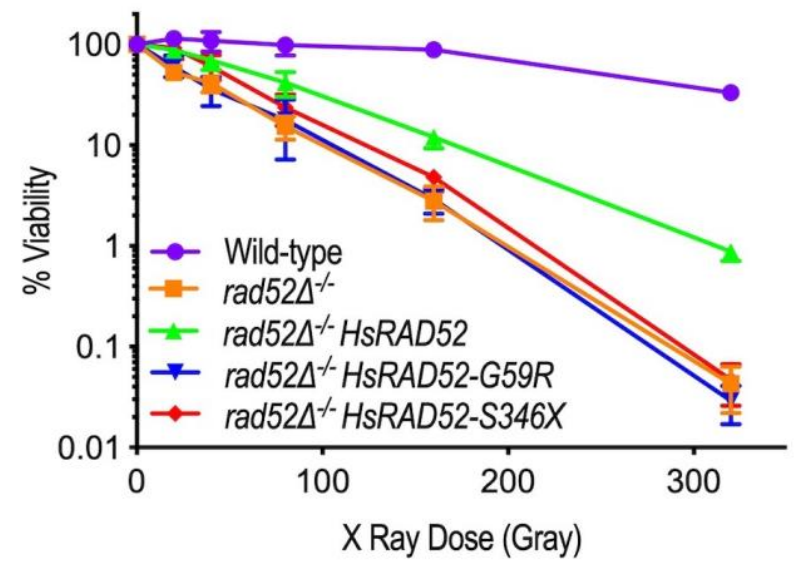

FLAG proteins do not function in the repair of IR-induced DSBs in budding yeast.

The HsRAD52-G59R and HsRAD52-S346X alleles cannot suppress the HRR defect of rad52 mutant cells

Budding yeast cells undergo mating type interconversion (MTI) by a highly efficient, programmed gene conversion event that proceeds by HRR of an $\mathrm{HO}$ endonuclease catalyzed DSB at the MAT locus on chromosome III using donor information from the flanking $H M L$ and $H M R$ loci (Figure 3A) [37-39]. In accordance with its role as the central HRR factor in budding yeast, mutations in RAD52 confer a dramatic loss in the efficiency of MTI [40]. Using a new assay for determining the frequency of MTI subsequent to galactose-induced expression of $\mathrm{HO}$ endonuclease, we observed a frequency near unity (59.6\%) in wild-type cells that was reduced 729 -fold in rad52 mutant cells (Figure 3B; Table S4). Expression of adh1::HsRAD52-FLAG in rad52 mutant cells suppressed the MTI defect by 69 -fold indicating that HsRAD52-FLAG possesses substantial functionality in this heterologous system (Figure 3B; Table S4). Importantly, adh1::HsRAD52-FLAG also suppressed the inability of rad52 mutant cells to repair a DSB at the HIS3 locus by gene conversion using an unlinked, defective copy of the HIS3 gene (Figure S1, Table S4) [29]. This indicates that HsRAD52 FLAG can support the repair of HO-catalyzed DSBs by HRR in multiple genomic contexts in budding yeast.

In contrast to expression of the adh1::HsRAD52-FLAG gene, we found that expression of the adh1::HsRAD52G59R-FLAG ( $\mathrm{p}=0.30)$ and adh1::HsRAD52-S346X-FLAG ( $\mathrm{p}=$ 0.31 ) mutant alleles failed to yield statistically significant changes in the frequencies of MTI in rad52 mutant strains (Figure 3B; Table S4). We also observed that these alleles conferred similar defects in the repair of a DSB at the HIS3 locus by HRR (Figure S1; Table S4). Combined with our observations that these alleles failed to suppress the IR sensitivity of rad52 $\%$ diploids (Table S4), these data indicate that the mutant HsRAD52-G59R-FLAG and HsRAD52-S346XFLAG proteins do not support the repair of HO-catalyzed or IR-induced DSBs by an HR mechanism that conserves genome structure.

FIGURE 2: The adh1::HsRAD52-G59R-FLAG and adh1::HsRAD52S346X-FLAG alleles fail to suppress the ionizing radiation sensitivity of $\mathrm{rad}^{2} \%$ mutant yeast cells. Cultures of wild-type (ABX3566), rad52\% (ABX3568), rad52\% adh1::HsRAD52-FLAG ${ }^{+/+}$ (ABX4130), rad52\% adh1::HsRAD52-G59R-FLAG $\%$ (ABX4129) and rad52\% adh1::HsRAD52-S346X-FLAG\% (ABX4131) yeast strains grown to mid-log phase were counted before being subjected to 20,40, 80, 160 and 320 Gy of X-ray radiation. Appropriate dilutions of unirradiated and irradiated cultures were plated onto solid YPD medium, incubated at $30^{\circ} \mathrm{C}$ for three days, and the resulting colonies counted. Percent viability was calculated by dividing the number of colonies arising on the plates by the number of cell bodies plated and multiplying by 100 . Mean percent survival was calculated for at least 10 independent cultures for each genotype. These values and $95 \%$ confidence intervals were plotted against levels of radiation exposure. 
A

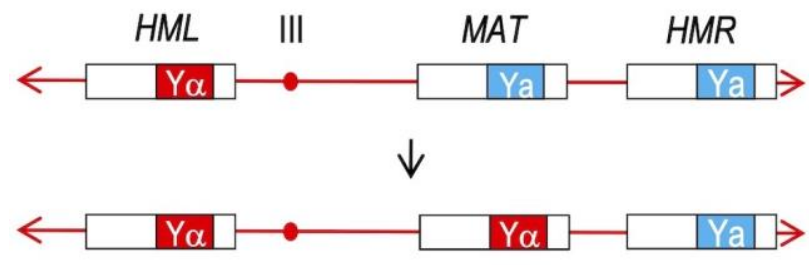

B

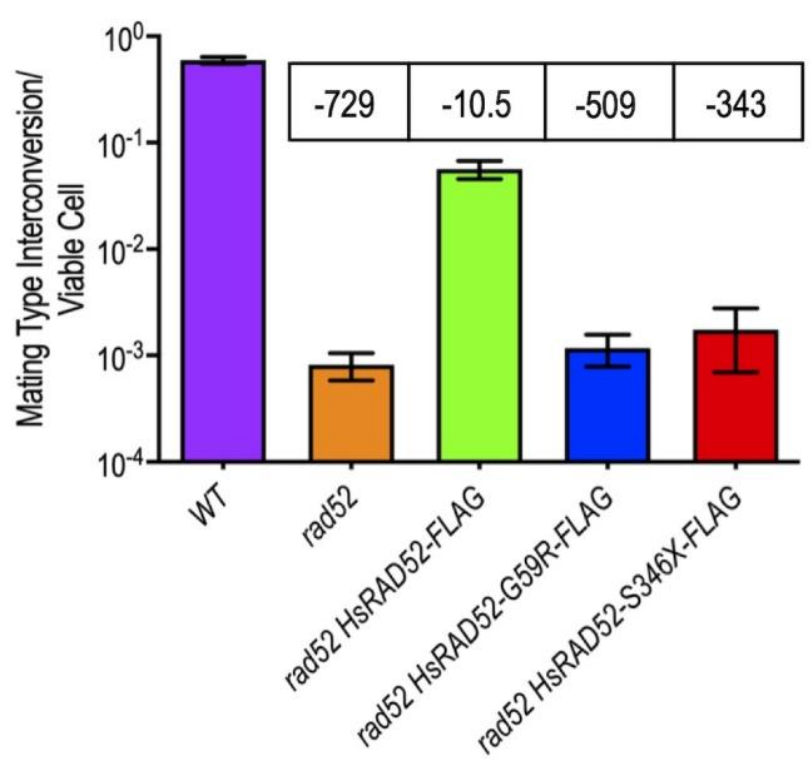

FIGURE 3: The adh1::HsRAD52-G59R-FLAG and adh1::HsRAD52S346X-FLAG alleles do not suppress the MTI defect of rad52 mutant yeast cells. (A) Cartoon depicting MTI. DSB formation by $\mathrm{HO}$ endonuclease cutting at the $\mathrm{HO}$ cut site on the centromere distal edge of the Ya sequence (blue box) at the MAT locus on chromosome III precipitates the exonucleolytic removal of "a" mating type information prior to its replacement by unidirectional transfer of " $\alpha$ " mating type information from the $Y \alpha$ sequence (red box) of the flanking, intact but silent $H M L$ locus. This results in the switching of the cell from the "a" mating type to the " $\alpha$ " mating type. (B) The adh1::HsRAD52-G59R-FLAG and adh1::HsRAD52S346X-FLAG alleles confer defects in MTI. Single colonies of haploid wild-type (ABX3817-15B), rad52 (ABX3817-7D), rad52 adh1::HsRAD52-FLAG (ABX3977-10C), rad52 adh1::HsRAD52G59R-FLAG (ABX3985-55B), and rad52 adh1::HsRAD52-S346X (ABX3994-18D) strains were used to inoculate at least 10 one milliliter YPGL cultures and grown overnight. Following a period of expression of $\mathrm{HO}$ endonuclease, appropriate dilutions were plated onto YPD medium, incubated for three days at $30^{\circ}$, and the number of colonies counted. Colonies were replica plated to fresh YPD plates, printed with a lawn of the haploid R113a mating type tester strain, printed plates incubated overnight at $30^{\circ} \mathrm{C}$ and then replica plated onto SD plates, which were incubated overnight at $30^{\circ} \mathrm{C}$. Frequencies of $\mathrm{MTI}$ were determined by dividing the number of diploid patches arising on the SD plates by the number of colonies counted on the original YPD plates. Mean frequencies of MTI and $95 \%$ confidence intervals were plotted against genotype. Fold differences below (-) the wild-type frequency of MTI for each strain are indicated in boxes above the bar for each mean frequency.
Wild-type and mutant alleles of HsRAD52 complement the loss of DSB repair by single-strand annealing (SSA) in rad52 mutant cells

The yeast and mammalian RAD52 proteins have been implicated in the repair of DSBs by SSA [41, 42], a nonconservative mechanism of repair that is genetically distinct from HRR [42-44]. Since the capacity of ScRad52 to propagate SSA has been localized to its $\mathrm{N}$-terminus [44, 45], the region of shared amino acid sequence homology with HsRAD52 (Figure S2) [46], we examined the ability of HsRAD52 to suppress the defect in DSB repair by SSA conferred by the rad52 mutation. We observed that repair of a $\mathrm{HO}$ endonuclease-catalyzed DSB at the HIS3 locus by recombination between duplicate 415 bp segments resulting in an intact HIS3 gene and deletion of the intervening $5 \mathrm{~kb}$ plasmid sequence was very efficient in wild-type cells, occurring in nearly $23 \%$ of survivors (Figure 4 ; Table S4). The frequency of DSB-DRR (direct repeat recombination) was reduced by nearly seven-fold in a rad52 mutant strain, confirming that DSB repair by SSA in budding yeast is largely dependent on ScRad52. Surprisingly, expression of adh1::HsRAD52-FLAG in a rad52 mutant strain completely suppressed this SSA defect, as frequencies of DSB-DRR were not statistically different from those observed in wildtype cells $(p=0.98)$. Further, the nearly 20 -fold reduced rate of spontaneous recombination between the $415 \mathrm{bp}$ direct repeats in rad52 mutant cells was also completely suppressed by the expression of adh1::HsRAD52-FLAG (Figure S3; Table S4). These results indicate that HsRAD52FLAG can fully replace ScRad52 for DSB-stimulated and spontaneous SSA in budding yeast cells.

In marked contrast to their effects on DSB repair by conservative HRR, expression of the adh1::HsRAD52-G59RFLAG and adh1::HsRAD52-S346X-FLAG mutant alleles in rad52 mutant strains completely suppressed their defects in DSB repair by non-conservative SSA (Figure 4; Table S4), as frequencies of DSB-DRR were not statistically different from those of wild-type ( $p \geq 0.17$ ), or rad52 adh1::HsRAD52-FLAG $(\mathrm{p} \geq 0.08)$ strains. These data indicate that the mutant HsRAD52-G59R-FLAG and HsRAD52S346X-FLAG proteins possess an essentially equivalent capacity to propagate DSB-stimulated SSA as ScRad52 or HsRAD52. Additionally, this establishes that the contributions of HsRAD52 to DSB repair by HRR and SSA are genetically distinct, and likely involve separate functions of the HsRAD52 protein in budding yeast cells.

\section{The HsRAD52-S346X allele supports formation of various HsRAD52 multimers}

Monomers of HsRAD52 can self-associate and form a homomeric ring structure that is thought to be of significance to its biochemical and cellular functions [47-50]. Having observed that adh1::HsRAD52-G59R-FLAG and adh1::HsRAD52-S346X-FLAG confer losses of function in IR resistance (Figure 2; Table S4) and DSB repair by HRR (Figure 3B; Figure S1; Table S4) in budding yeast, we investigated the ability of the HsRAD52-G59R and HsRAD52S346X mutant proteins to associate and form multimers using the yeast two hybrid $(\mathrm{Y} 2 \mathrm{H})$ and gel filtration analyses. 
A

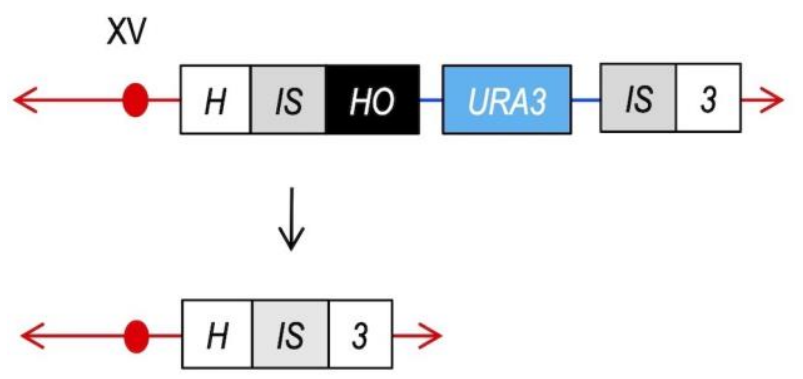

B

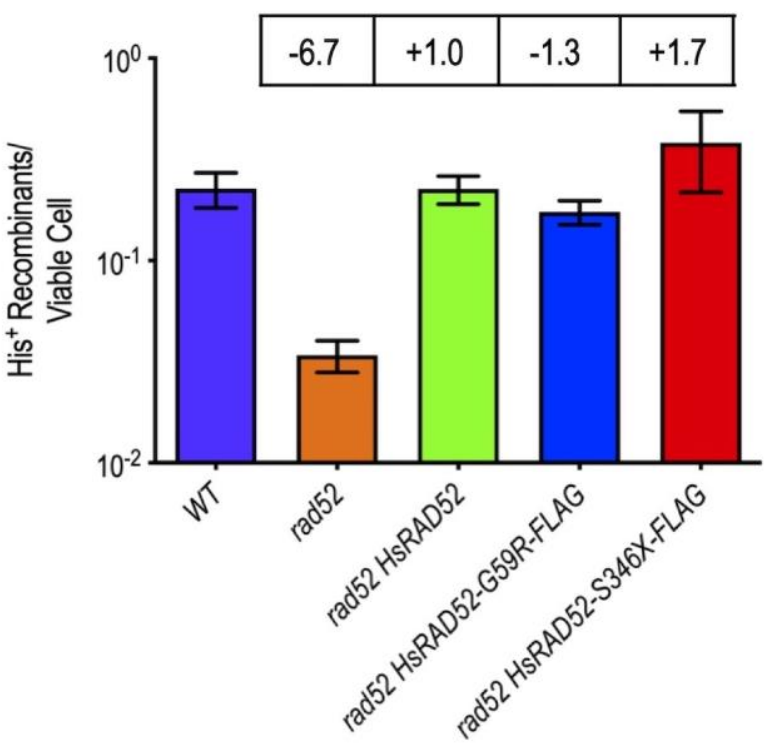

FIGURE 4: The adh1::HsRAD52-G59R-FLAG and adh1::HsRAD52S346X-FLAG alleles complement the loss of DSB repair by SSA in rad52 mutant yeast cells. (A) Cartoon depicting DSB repair by recombination between non-tandem direct repeats. At the HIS3 locus on chromosome XV, DSB formation by $\mathrm{HO}$ endonuclease cutting at a $\mathrm{HO}$ cut site (black box) inserted at the right edge of the left duplication of a segment of the HIS3 coding sequence (left gray IS box) initiates bidirectional exonucleolytic processing. Processing reveals complementary single-stranded sequences at the left and right repeats (left and right gray IS boxes) that anneal, creating non-homologous tails whose removal deletes intervening plasmid sequences (blue line and aqua URA3 marker box) enroute to creating an intact HIS3 gene. (B) The adh1::HsRAD52-G59RFLAG and adh1::HsRAD52-S346X-FLAG alleles complement the defects in DSB repair by DRR. Single colonies of haploid wild-type (ABM325), rad52 (ABM326), rad52 adh1::HsRAD52 (ABM507), rad52 adh1::HsRAD52-G59R-FLAG (ABX3970-88A), and rad52 adh1::HsRAD52-S346X (ABX3975-15A) strains were used to inoculate at least 10 one milliliter YPGL cultures and grown overnight. After a period of expression of $\mathrm{HO}$ endonuclease, appropriate dilutions were plated onto solid YPD medium to determine viability, and onto medium lacking histidine to select for recombinants. Following incubation for three days at $30^{\circ}$ colonies were counted and frequencies of DRR determined by dividing the number of $\mathrm{His}^{+}$recombinants by the number of viable cells plated. Mean frequencies of DRR and 95\% confidence intervals were plotted against genotype. Fold differences above $(+)$ and below (-) wildtype are indicated in the boxes above the bar for each mean frequency.
We observed levels of $\beta$-galactosidase activity in the extracts of yeast cells transformed with plasmids expressing the HsRAD52-Gal4 DNA binding domain and HsRAD52-Gal4 transcription activation domain fusion proteins (Table S2) that were substantially ( 80 -fold) above background (Figure S4; Table S5). These results are consistent with the selfassociation of HsRAD52 monomers in budding yeast cells. Expression of the HsRAD52-G59R and HsRAD52-S346X mutant fusion proteins produced levels of $\beta$-galactosidase activity that were not statistically different from those observed with the wild-type HsRAD52 fusion proteins ( $p \geq$ 0.51), indicating that HsRAD52-G59R and HsRAD52-S346X possess a capacity for self-association that is comparable to that of HsRAD52.

While pair-wise interactions between HsRAD52 monomers are very likely to contribute to the formation of homo-heptameric HsRAD52 ring structures, they may not be the sole determinants governing the formation and stabilization of these structures. In order to define the relative capacities of wild-type and mutant HsRAD52 proteins to form higher order multimers, we performed gel filtration chromatography to define their hydrodynamic properties. We utilized peptides containing the N-terminal 212 amino acids (1-212) of HsRAD52 and HsRAD52-G59R for these analyses, as HsRAD52 $2_{1-212}$ contains the domain required for self-association [48], forms undecameric rings in vitro [51, 52], and is substantially more stable than the full-length protein. The HsRAD52 $2_{1-212}$ and HsRAD52-G59R ${ }_{1-212}$ multimers displayed virtually identical column elution profiles, with peaks centered at an estimated molecular weight of $360 \mathrm{kDa}$, which could approximate a mass equivalent to thirteen to fourteen subunits of the $27 \mathrm{kDa} H s R A D 52_{(1-212)}$ monomers (Figure 5). These results suggest that the HsRAD52-G59R mutation does not confer marked changes in the quaternary structure of HsRAD52. In contrast, HsRAD52-S346X displayed an elution profile consistent with two multimer of relatively equal abundance; one with an estimated molecular weight of $257 \mathrm{kDa}$ that approximates to a hexameric multimer of $41 \mathrm{kDa}$ monomers, and a second with an estimated molecular weight of $335 \mathrm{kDa}$ that would be suggestive of an octamer.

The HsRAD52-G59R and HsRAD52-S346X alleles alter interactions between HsRAD52 and recombination substrates during DSB repair by HRR

The function of HsRAD52 in DSB repair by HRR in budding yeast cells was previously correlated with its progressive accumulation at the genomic DSB as documented by ChIP [29]. Accordingly, we used ChIP to study the association of HsRAD52-FLAG with the MATa locus during MTI in strains where the HMR locus was deleted to prevent contamination by signal acquired from the "a" information at that locus (Figure 6A). We observed that following DSB formation, HsRAD52-FLAG accumulated at the MAT locus in rad52 adh1::HsRAD52-FLAG cells, peaking at 14.6-fold enrichment at six hours (Figure 6B). The HsRAD52-S346XFLAG mutant protein associated with the MAT locus with kinetics and at levels that were very similar to those of HsRAD52-FLAG $(p=0.22)$, whereas the HsRAD52-G59R- 


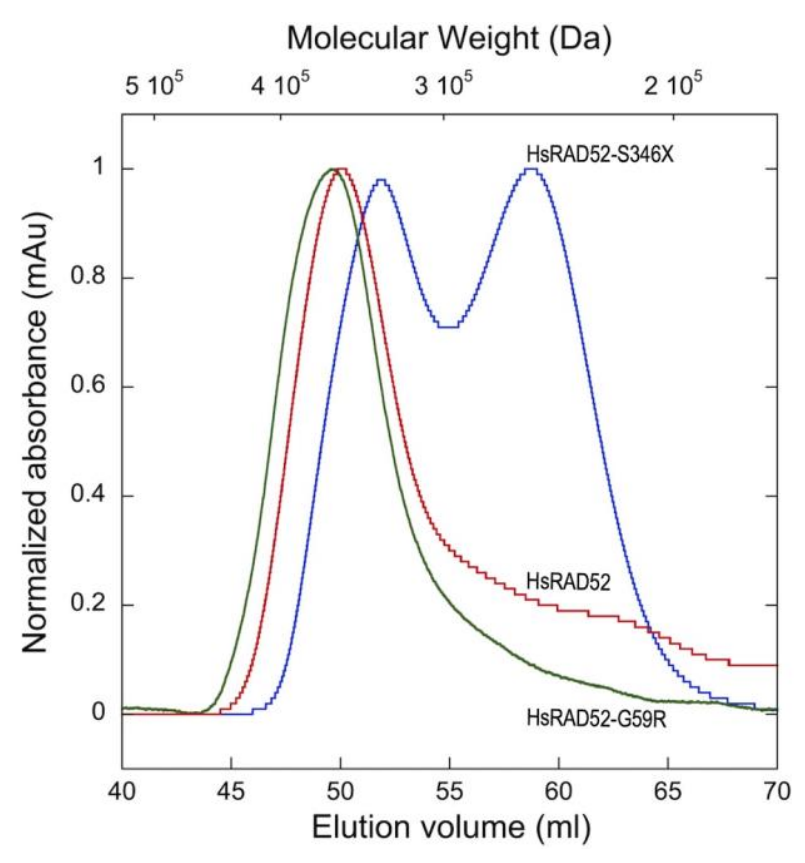

FIGURE 5: HsRAD52-S346X alters the in-solution oligomerization state of HsRAD52. Superdex 200 size exclusion elution profiles are shown for HsRAD52 1 -212, HsRAD52-G59R ${ }_{1-212}$, and HsRAD52S346X. HsRAD52 ${ }_{1-212}$ (red trace) eluted as a single peak at $50.0 \mathrm{ml}$, with an estimated size of $360 \mathrm{kDa}$. HsRAD52-G59R ${ }_{1-212}$ (green trace) eluted as a single peak at $49.6 \mathrm{ml}$ with an estimated size of 366kDa. HsRAD52-S346X (blue trace) eluted as two peaks; the first peak eluted at $51.9 \mathrm{ml}$ with an estimated size of $335 \mathrm{kDa}$, and the second peak eluted at $58.7 \mathrm{ml}$ with an estimated size of $257 \mathrm{kDa}$. (mAU = milliabsorbance unit).

FLAG mutant protein displayed similar kinetics, but a level of accumulation at six hours that was two-fold lower $(p=$ 0.0002 ) than that of HsRAD52. Interestingly, ScRad52-FLAG also accumulated at the MAT locus with similar kinetics to those of HsRAD52-FLAG, but reached a nearly five-fold greater level of enrichment at six hours.

Molecular models for MTI propose that the HRR apparatus assembles at the MAT locus subsequent to DSB formation after which there is a search for and pairing with intact homologous sequences at the flanking $H M L$ or $H M R$ loci [39]. Accordingly, previously published experiments indicated that ScRad52 associates sequentially with MAT and $H M L$ (or HMR) [53]. Our ChIP data indicated that ScRad52-FLAG associates with the MAT and HML loci with distinct kinetics. ScRad52-FLAG had accumulated 15-fold at MAT 2 hours after DSB formation, which was less than 4-fold below the level observed at 6 hours (Figure 6B). In contrast, ScRad52-FLAG had accumulated only 2.8-fold at $H M L$ after 2 hours before reaching a peak accumulation of 12.8-fold at 6 hours (Figure $6 \mathrm{C}$ ). These data are consistent with ScRad52-FLAG partaking in separate interactions with the MATa recipient and $H M L$ donor substrates during MTI in our strains.

Like ScRad52-FLAG, HsRAD52-FLAG displayed distinct kinetics of association with the MAT and HML loci subsequent to DSB formation, accumulating rapidly at MAT (Fig- ure 6B) and slowly at $H M L$ (Figure 6C) in rad52 adh1::HsRAD52-FLAG strains, and consistent with HsRAD52-FLAG interacting separately with MAT and $H M L$ during MTI. In notable contrast to its accumulation at MAT, HsRAD52-FLAG displayed limited accumulation at $H M L$ that was only significantly over background $(p=0.0017)$ at six hours (Figure 6C). This peak, 2.2-fold level of accumulation of HsRAD52-FLAG at HML was 5.8-fold lower than the peak accumulation of ScRad52-FLAG, indicative of substantially more restricted association. The accumulation of the HsRAD52-G59R-FLAG and HsRAD52-S346X-FLAG mutant proteins at $H M L$ were not significantly above background $(p \geq 0.18)$ at any time point, and were significantly below that of HsRAD52-FLAG at its six hour peak of accumulation $(p \leq 0.05)$. These results are consistent with both HsRAD52G59R-FLAG and HsRAD52-S346X-FLAG possessing defects in the ability to associate with $H M L$ during MTI.

The HsRAD52-G59R and HsRAD52-S346X alleles do not support repair synthesis in rad52 mutant cells

The prevailing model for MTI proposes that following DSB formation at the MAT locus, MAT sequences invade intact, homologous sequences at the HML locus forming a heteroduplex that is extended by repair synthesis, which replaces information lost from MAT with information from $H M L$ (Figure 7A) [39]. Previous studies found that repair synthesis reached near maximum levels by two hours after DSB formation at the MAT locus [54]. Similarly, our studies with RAD52-FLAG yeast strains revealed a seven-fold level of accumulation of the repair synthesis intermediate at two hours that was not significantly different at subsequent time points ( $p \geq 0.27$ ) (Figure 7B). These results are consistent with rapid interaction between MAT and HML genomic sequences following DSB formation at the MAT locus.

In marked contrast to the RAD52-FLAG yeast strains, the rad52 adh1::HsRAD52-FLAG strains displayed slow accumulation of the repair synthesis intermediate after DSB formation at the MAT locus, with levels that were not significantly above background at two hours ( $p=0.64$ ), and increasing to a peak accumulation of 8.8-fold at six hours (Figure 7B). These data indicate that ScRad52-FLAG supports a more rapid interaction between $M A T$ and $H M L$ genomic sequences, and extension of the heteroduplex by repair synthesis than HsRAD52-FLAG. Importantly, the repair synthesis intermediate did not accumulate significantly $(p \geq 0.16)$ throughout the time course in either the rad52 adh1::HsRAD52-G59R-FLAG or rad52 adh1::HsRAD52S346X-FLAG mutant strains, consistent with HsRAD52G59R and HsRAD52-S346X failing to support interaction between MAT and HML genomic sequences, and/or extension of the heteroduplex by repair synthesis. These results are consistent with the differential abilities of the adh1::HsRAD52-FLAG, adh1::HsRAD52-G59R-FLAG and adh1::HsRAD52-S346X-FLAG alleles to support MTI in rad52 mutant budding yeast cells (Figure 3B; Table 54 ). 
A

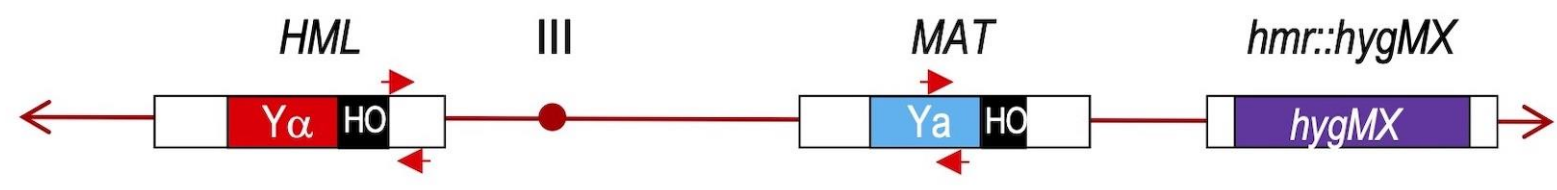

B

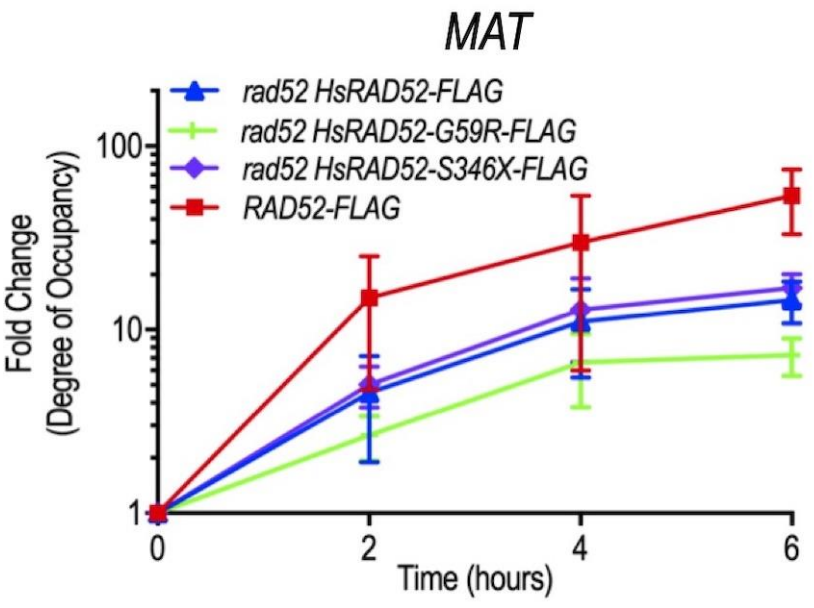

C

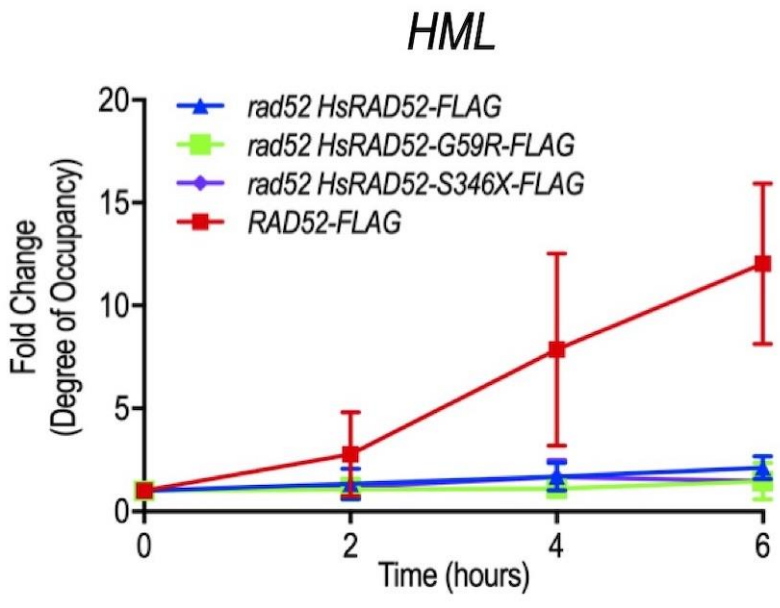

FIGURE 6: The adh1::HsRAD52-G59R-FLAG and adh1::HsRAD52-S346X-FLAG alleles have differential effects on the interaction of HsRAD52 with the MAT and HML loci during MTI. (A) Cartoon depicting substrates for MTI and location of primers used for quantitation of immunoprecipitated genomic sequences. Following DSB formation by $\mathrm{HO}$ endonuclease at the HO cut site (black box) at the MAT locus on chromosome III, exonucleolytic processing results in the accumulation of ssDNA in flanking sequences. ScRad52-FLAG, or HsRAD52-FLAG progressively associate with the ssDNA. This association facilitates retention of the DNA sequences by ChIP, which are quantitated by qPCR using MATa recipient primers (red arrows; Table S3) complementary to a 110 bp sequence laying 500 bp upstream from the DSB at MAT. Deletion of the $H M R$ locus and replacement with a hygMX marker (purple box) removes additional complementary sequences from the genome. After association with ssDNA at MAT, the HRR apparatus facilitates a search for homologous genomic sequences that gives rise to heteroduplex formation with the intact $H M L$ locus. The sequences that lay proximal to the HO cut site at the border of $Y \alpha$ (red box) are the putative initial location for heteroduplex formation. Association of ScRad52-FLAG or HsRAD52-FLAG with the heteroduplex results in retention of these sequences by ChIP, and their quantitation is done by qPCR using HML $\alpha$ donor primers (red arrows; Table S3) complementary to a 187 bp sequence laying $67 \mathrm{bp}$ downstream from the HO cut site sequence at HML. (B) ScRad52-FLAG and HsRAD52-FLAG display similar kinetics of association with sequences at the MAT locus after DSB formation. Single colonies of wild-type (ABX3961-4C), rad52 (ABX3943-3B), rad52 adh1::HsRAD52-FLAG (ABX3977-10C), rad52 adh1::HsRAD52-G59R-FLAG (ABX3985-55B), and rad52 adh1::HsRAD52-S346X-FLAG (ABX3994$18 \mathrm{D})$ strains carrying $\mathrm{MTI}$ assay components were used to establish cultures from which aliquots were collected at various times before and after DSB formation at the MAT locus by HO endonuclease. Whole cell extracts were prepared, subjected to ChIP using anti-FLAG antibody and the immunoprecipitated DNA from the MAT (experimental) and SAM1 (control) loci quantitated by qPCR. Fold changes in degree of occupancy of the FLAG-tagged proteins relative to those observed before DSB formation were normalized to a control strain lacking FLAGtagged proteins (ABX3933-46C). Mean fold changes from at least eight determinations using DNA collected from at least three independent time courses, and standard deviations were plotted against elapsed time after initiation of DSB formation. (C) HsRAD52-G59R-FLAG and HsRAD52-S346X-FLAG display defects in association with the HMR locus during MTI. Same as above except immunoprecipitated DNA from the $H M R$ locus (experimental) was quantitated by qPCR.

\section{DISCUSSION}

The structure [51, 55-57] and several biochemical activities [58-61] of the RAD52 protein have been conserved from bacteriophage to humans. However, its cellular function has changed substantially through phylogenesis; it plays an integral role in the canonical HRR apparatus in budding yeast [62-64], but a separate role in mammals [19-21]. In support of this diversification, we previously demonstrated that HsRAD52 possesses the distinct ability to promote repair of DSBs by conservative HRR in budding yeast independently from the canonical HRR apparatus [29], which may be similar to its function in mammalian cells $[20,22$ 65].

The current study demonstrates that expression of adh1::HsRAD52-FLAG in rad52 mutant budding yeast cells substantially suppressed the X-ray sensitivity (Figure 2; Table S4) and MTI defects (Figure 3; Table S4). The 70-fold suppression of the rad52 MTI defect by adh1::HsRAD52FLAG is of particular interest as it demonstrated that HsRAD52-FLAG can function effectively within a highly specialized, heterologous system for DSB repair $[66,67]$, even though it does not associate with the endogenous HRR 
A

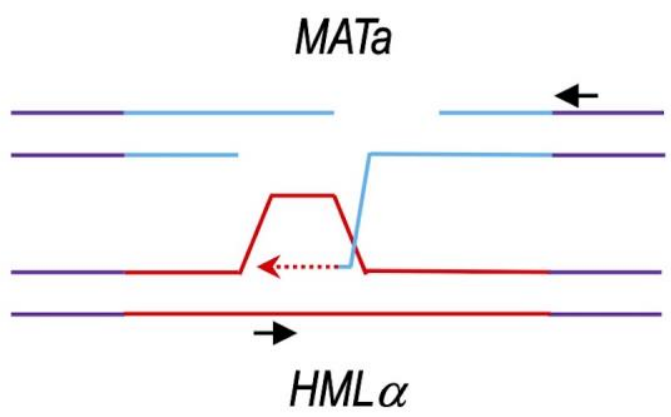

B

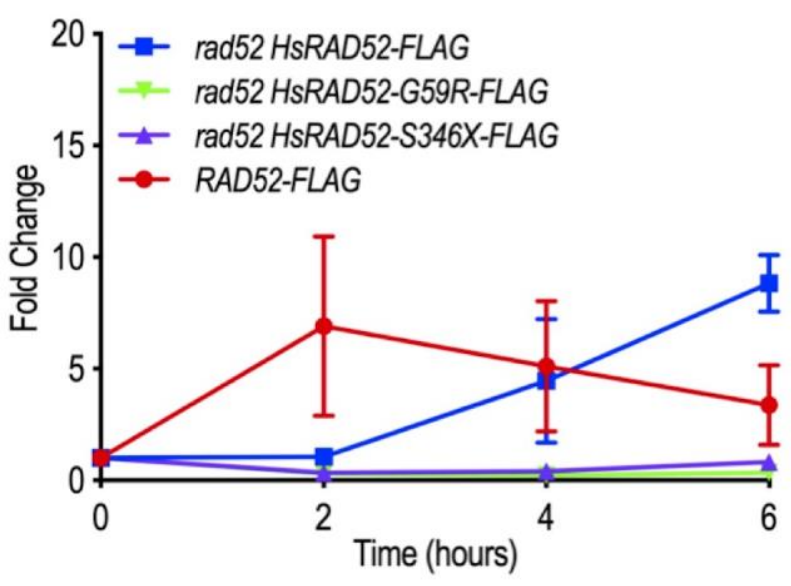

FIGURE 7: The adh1::HsRAD52-G59R-FLAG and adh1::HsRAD52S346X-FLAG alleles confer defects in repair synthesis during MTI. (A) Cartoon depicting extension by repair DNA synthesis of putative heteroduplex formed upon association between sequences at the $M A T$ and $H M L$ loci during MTI. Following DSB formation at the MAT locus (blue lines) exonucleolytic processing creates ssDNA onto which ScRad52-FLAG and HsRAD52-FLAG are proposed to bind. A search for homology putatively results in formation of a heteroduplex linking homologous sequences at the broken MAT locus with sequences at the intact $H M L$ locus (solid red lines). Extension of the heteroduplex by DNA repair synthesis (dotted red line) copies " $\alpha$ " information from $H M L$ that replaces "a" information lost from MAT, ultimately resulting in a change from MATa to MAT $\alpha$. Repair synthesis ultimately covalently joins " $\alpha$ " information from $H M L$ with sequences downstream from the MAT locus, which can be detected by PCR using the depicted primers (black arrows). (B) Repair synthesis was defective in rad52 adh1::HsRAD52-G59R-FLAG and rad52 adh1::HsRAD52-S346X-FLAG mutant cells. Genomic DNA was collected before immunoprecipitation from the same wild-type (ABX3961-4C), rad52 adh1::HsRAD52-FLAG (ABX3977-10C), rad52 adh1::HsRAD52-G59R-FLAG (ABX3985-55B), and rad52 adh1::HsRAD52-S346X-FLAG (ABX3994-18D) cultures used for the ChIP analyses described above (Fig 6). Extension by repair synthesis of the putative $M A T / H M L$ heteroduplex was quantitated by semiquantitative end-point PCR using the primers depicted in panel A. DNA from the intact SAM1 locus was also quantitated as a signal for normalization. PCR products were separated on agarose gels, stained with ethidium bromide and band intensities were quantified using ImageJ. Normalized mean ratios and corresponding standard deviations from three independent time courses were calculated by dividing signal obtained from repair synthesis with the signal from the SAM1 control. All time point values were then normalized to the signal obtained before DSB formation ( $T=0 \mathrm{hrs}$ ) and plotted against elapsed time after DSB formation. machinery [29]. The current studies also indicate that HsRAD52-FLAG and ScRad52-FLAG interacted similarly with recipient sequences at the MAT locus (Figure 6B; Table S4), but distinctly with donor sequences at the HML locus (Figure $6 \mathrm{C}$ ), and that HsRAD52-FLAG supported a slower progression to the repair synthesis step of MTI (Figure 7B; Table S4). These results extend our genetic and molecular analyses of HsRAD52 function in conservative HRR in budding yeast, and further confirm that HsRAD52-dependent HRR proceeds through steps and intermediates that are similar to those of ScRad52-dependent HRR, but with distinct kinetics and as part of a distinct apparatus.

In contrast to its incomplete suppression of the MTI defect in rad52 mutant cells, expression of adh1::HsRAD52FLAG fully complemented the SSA defect (Figure 4B; Table S4), suggesting that HsRAD52-FLAG plays distinct roles in the repair of DSBs by HRR and SSA in budding yeast cells. The capacity of HsRAD52-FLAG to replace ScRad52 in SSA is consistent with previous studies that mapped the SSA function of ScRad52 to its N-terminus [29, 44], the area of greatest shared amino acid sequence homology with HsRAD52 (Figure S2). Consequently, the relationship between the primary structure of this region of HsRAD52 and its ability to support SSA in budding yeast will be a subject of continued investigation. Additionally, the capacity of HsRAD52-FLAG to act as a replacement for ScRad52 in SSA suggests that it works in concert with other factors important for SSA in yeast [68-76], which will also be investigated in the future.

Several naturally occurring variants of human HRR genes have been shown to confer defects in HRR and cancer susceptibility [77-79], linking HRR to disease resistance. Accordingly, we investigated the effects on DSB repair in budding yeast of two HsRAD52 variants identified in African-American women with breast cancer, HSRAD52-G59R and HsRAD52-S346X; the latter recently reported to attenuate the pathogenicity of $B R C A 2$ mutations in breast cancer [30]. The HsRAD52-G59R variant confers a change in an $\mathrm{N}$-terminal amino acid of HsRAD52 that is conserved between the human and yeast homologs (Figure S2), while HsRAD52-S346X deletes 72 amino acids from the C-terminus of HsRAD52, a region that shares little sequence similarity with the yeast homolog. Interestingly, despite their affecting either conserved or divergent regions of the primary sequence of HsRAD52, the variants exerted nearly identical effects on DSB repair at the genetic and molecular levels in budding yeast. Both mutations essentially ablated the capacity of HsRAD52 to support the repair of $X$ ray- and $\mathrm{HO}$ endonuclease-generated DSBs by conservative HRR (Figures 2 and 3; Table S4). In striking contrast, neither mutation had a significant effect on the repair of a HO-catalyzed DSB by SSA (Figure 4; Table S4), establishing that the HsRAD52-G59R and HsRAD52-S346X are separation-of-function mutations. This indicates that HsRAD52-FLAG supports DSB repair by conservative HRR and non-conservative SSA through distinct mechanisms in budding yeast. Interestingly, expression of the HsRAD52S346X allele in $R A D 52^{\%}$ mutant mouse embryonic stem cells was recently shown to support a two-fold lower level 
of DSB repair by SSA than the HSRAD52 allele [30], suggesting that the function of HsRAD52 in SSA in yeast and mouse cells might be different. However, this reduced level of SSA correlated with reduced nuclear localization of HsRAD52S346X, which is the likely outcome of loss of the nuclear localization signal from the C-terminus [80], indicating that the reduction in SSA may not be due to any loss of biochemical activity.

We previously employed ChIP to study the effects of HR gene mutations on HR protein function during DSB repair in budding yeast [81], and have used this approach to examine the effects of the HsRAD52-G59R-FLAG and HsRAD52-S346X-FLAG alleles on the association of HsRAD52-FLAG with the MAT and HML loci during MTI. Following DSB formation at the MAT locus, the HsRAD52FLAG, HsRAD52-G59R-FLAG and HsRAD52-S346X-FLAG proteins associated with the MAT locus with similar kinetics. However, the peak level of accumulation of HsRAD52G59R-FLAG was two-fold lower than that of HsRAD52-FLAG $(p=0.002$ ) (Figure 6B; Table S4). This reduction might result from the change of a small, uncharged glycine at one edge of the putative ssDNA binding cleft in HsRAD52 to a bulky, positively charged arginine [51], which could impede free passage of negatively charged ssDNA into the binding cleft. The extent to which the reduced association of HsRAD52-G59R-FLAG with the DSB at MAT contributes to the reduced frequencies of MTI in rad52 adh1::HsRAD52G595R-FLAG cells is unclear (Figure 3; Table S4). However, it is interesting to note that a reduction in association of HsRAD52-G59R-FLAG with a DSB might be expected to impact repair by SSA, however no significant effect was observed $(p=0.23$ ) (Figure 4; Table S4). This indicates that even the reduced capacity of HsRAD52-G59R-FLAG to associate with DSBs is sufficient for essentially wild-type levels of repair by SSA. This is particularly interesting as the peak level of association of ScRad52-FLAG with the DSB at MAT was five-fold higher than that of HsRAD52-FLAG ( $p<$ 0.0001 ) (Figure 6), suggesting that levels of association of ScRad52-FLAG and HsRAD52-FLAG with DSBs are well in excess of those necessary for efficient repair by SSA in budding yeast.

Our examination of the association of ScRad52-FLAG with the MAT and $H M L$ loci revealed that it accumulated more slowly at $H M L$ (Figure 6B and C), which was previously interpreted as evidence of sequential association with MAT and HML during MTI $[53,54]$. ScRad52-FLAG ultimately reached a substantial 12.6-fold peak level of accumulation at $H M L$ consistent with frequencies of MTI of near unity in wild-type yeast cells (Figure 3B; Table S4). Like ScRad52-FLAG, HsRAD52-FLAG accumulated rapidly at MAT after DSB formation, but accumulated much more slowly and to much lower peak levels at HML. This could be due to: a) limitations in the sensitivity of the qPCR method used to detect this association in rad52 adh1::HsRAD52-FLAG cells where frequencies of MTI were 10-fold lower than in wild-type cells (Figure 3B; Table S4); or b) indicative of HsRAD52-FLAG-dependent MTI utilizing the sequences at $H M L$ in a distinct manner at the molecular level. The differential association of HSRAD52 with the MAT and HML loci is consistent with its three-order-of-magnitude greater affinity for ssDNA than dsDNA [82]. Importantly, like ScRad52-FLAG, HsRAD52-FLAG supported the extension by repair synthesis of heteroduplex most likely formed as part of a ternary complex with the MAT and HML loci (Figure 7). This indicates that whatever the nature of association between HsRAD52-FLAG and HML, repair of the DSB at MAT during MTI in rad52 adh1::HsRAD52-FLAG cells involved the use of $H M L$ sequences as the donor template. Alternative hypotheses for how HsRAD52 may support such heteroduplex formation, including increasing the flexibility of dsDNA prior to intercalation with ssDNA [82], transient unpairing of dsDNA in advance of base-pairing with ssDNA [83], or formation of a dynamic, sliding heteroduplex [84] will be investigated further.

We observed that the impact of the adh1::HsRAD52G59R-FLAG and adh1::HsRAD52-S346X-FLAG alleles on association of HsRAD52-FLAG with the MAT locus during $\mathrm{MTI}$ was modest or undetectable (Figure 6B). However, both alleles resulted in the failure of HSRAD52-FLAG to associate with the $H M L$ locus (Figure $6 \mathrm{C}$ ) and failure to extend MAT-HML heteroduplex by repair synthesis (Figure 7) in rad52 mutant cells. This is consistent with the observation that $\mathrm{X}$-ray resistance and frequencies of $\mathrm{MTI}$ in rad52 adh1::HsRAD52-G59R-FLAG and rad52 adh1::HsRAD52-S346X-FLAG mutant cells were not significantly different from those of rad52 mutant cells (Figures 2 and 3B; Table S4). This also suggests that these cells are blocked at a step in the repair of DSBs by conservative HRR that occurs between association of HsRAD52-FLAG with ssDNA sequences adjacent to the DSB, and the interaction of the broken sequences with intact, homologous donor sequences in the genome. Intriguingly, the glycine at position 59 in the primary amino acid sequence of HsRAD52 lays at the apex of a loop that extends to the outer periphery of the "domed cap" observed in the crystal structure formed by a multimer of HsRAD52 N-terminal half peptides, an area thought to be involved in binding to dsDNA [51,56]. This suggests that changing the glycine to an arginine mildly disrupts interaction with ssDNA adjacent to the DSB, but completely blocks interaction with intact double-stranded donor sequences and any subsequent steps in HRR. The potential impact on the behavior of HsRAD52 of deleting 72 amino acids from the C-terminus is more difficult to contemplate as this region has not been crystallized and contains few recognizable motifs. However, the deletion led to the formation of various HsRAD52 multimers in contrast to the single, heptameric form reported previously for the full-length wild-type protein (Figure 5) [85]. Previous research showed that the C-terminal half of HsRAD52 can direct self-association separately from the phylogenetically conserved N-terminal self-association domain [48, 86], suggesting that deletion of the C-terminal 72 amino acids may disrupt this signal and affect multimerization. The impact of both structural changes on the capacity of HsRAD52 to associate with dsDNA will be a focus of future investigations.

The observation that HsRAD52-S346X both protects against breast cancer in carriers of pathogenic BRCA2 mu- 
tations [30], and confers a loss of HsRAD52-dependent DSB repair by HRR in budding yeast cells suggests that a similar DNA repair defect may contribute to the synthetic lethality thought to promote the protective effect in humans. Interestingly, HsRAD52-S346X also confers a defect in DSB repair by SSA in mammalian cells, suggesting that this variant may precipitate multiple DSB repair defects that impinge upon the survivorship of BRCA2-defective cells [30]. Additionally, HsRAD52 has recently been shown to support other HR-dependent functions in mammalian cells $[84,87]$ that might also support the survival of BRCA2-defective cells, and whose diminution by HsRAD52-S346X could negatively affect viability. Further study of the effects of HsRAD52-S346X on these functions in human cells should clarify how HsRAD52 contributes to the survival of BRCA2defective cells.

The current study suggests that our model system for examining HsRAD52 function in budding yeast cells can be used to further explore the relationship between the structure of HsRAD52 and its function in DSB repair by HRR. This novel approach may unlock a diversity of strategies for selectively killing HRR-defective cancers [21]. The utility of this system in defining defects conferred by HsRAD52 lossof-function mutations at the molecular level indicates that it could prove useful for examining the mechanism of action of small molecule inhibitors of HsRAD52 at the molecular level in living cells. Inhibitors of HsRAD52-dependent MTI might be potent and selective inhibitors of HRRdeficient cancers by themselves or in combination with other therapeutic strategies, such as the use of inhibitors of poly (ADP ribose) polymerase [88] or ionizing radiation. The growing significance of the HRR-defective phenotype in a broad array of cancers [89-92] suggests that such inhibitors could play an expanding role in future cancer treatment regimens.

\section{MATERIALS AND METHODS}

\section{Yeast strains}

All strains used (Table S1) were isogenic and constructed, maintained and grown using established procedures [93].

\section{Plasmids}

Construction and amplification of plasmids (Table S2) utilized established techniques [94]. The plasmids utilized in the yeast two-hybrid analysis were constructed using pGBT9, a vector containing a TRP1 selectable marker and sequences that encode the DNA binding domain of the yeast Gal4 protein, and pGAD424, which contains a LEU2 selectable marker and sequences encoding the transcription activation domain of Gal4 (Clontech, Mountain View, CA, USA). The plasmids pGBT9HsRAD52 and pGAD424-HsRAD52 express fusions of the Gal4 DNA binding domain and the Gal4 transcriptional activation domain to the N-terminus of wild-type HsRAD52, respectively, and were described in a previous study [29]. Plasmids pGBT9HsRAD52-G59R and pGBT9-HsRAD52-S346X were constructed from pGBT9-HsRAD52 and express fusions of the mutant HsRAD52-G59R and HsRAD52-S346X proteins to the Gal4 DNA binding domain. Plasmids pLAY700 and pLAY701 were constructed from pGAD424-HsRAD52 and express fusions of HsRAD52-G59R and HsRAD52-S346X to the Gal4 transcription- al activation domain. The plasmids used to express wild-type and mutant HsRAD52 proteins for native complex size determination were constructed using $\mathrm{pET} 28 \mathrm{~b}$, which contains a kanamycin resistance marker and sequences for the bacterial expression of proteins tagged at the $\mathrm{N}$-terminus with six, tandem histidine residues (Novagen/Merck, Darmstadt, GDR). The plasmids pLAY855, pLAY970 and pLAY971 were constructed from $\mathrm{pET} 28 \mathrm{~b}$ and were used to express $\mathrm{N}$-terminally 6-Histagged wild-type HsRAD52, and mutant HsRAD52-S346X and HsRAD52-G59R respectively.

\section{Cellular protein detection}

Wild-type and mutant HsRAD52-FLAG proteins were detected using Western blot analysis as described previously [29]. Whole cell extracts of cells of the appropriate genotype were prepared by glass bead disruption, and the proteins separated on acrylamide gels before transfer to nylon membranes (Imobilon-P PVDF, Millipore Sigma, St. Louis, MO, US). Proteins were detected with anti-FLAG M2 (Millipore Sigma) and antiGAPDH (Aviva Systems Biology, San Diego, CA, USA) primary antibodies, goat anti-mouse HRP-conjugated secondary antibody (Thermo Fisher Scientific, Waltham, MA, USA), chemiluminescent signal generation (SuperSignal West Femto Maximum Sensitivity Substrate, Thermo Fisher Scientific), and visualization on X-ray film.

\section{Determining levels of ionizing radiation resistance}

A minimum of 10 five milliliter YPD ( $1 \%$ yeast extract, $2 \%$ bacto peptone, $2 \%$ dextrose) cultures were inoculated with single colonies of selected yeast strains, grown to a density of $1-3 x$ $10^{6}$ cells $/ \mathrm{ml}$, washed, resuspended in $5 \mathrm{ml}$ of distilled water, and cells counted by hemacytometer. Specified doses of ionizing radiation from a Xrad320 Xray irradiator (Precision X-Ray, North Branford, CT, USA) were applied to aliquots of cell suspension before appropriate dilutions were plated onto YPD medium, and the number of colonies counted after incubation at $30^{\circ} \mathrm{C}$ for three days. Percent viability was determined by dividing the number of colonies by the number of cells plated and multiplying by $100.95 \%$ confidence intervals and t-test values were calculated with Prism (GraphPad, San Diego, CA, USA).

\section{Mating type interconversion (MTI) frequency determination} A minimum of 10 one milliliter YPGL (1\% yeast extract, $2 \%$ bacto peptone, $3 \%$ glycerol, $3 \%$ lactate) cultures were inoculated with single colonies of selected MATa yeast strains with a galactose-inducible $\mathrm{HO}$ endonuclease gene inserted into the TRP1 locus (trp1::GAL-HO-kanMX) with or without a deletion of the HMR locus ( $h m r:: h y g M X)$, and grown overnight at $30^{\circ} \mathrm{C}$ to a density of $1-2 \times 10^{7}$ cells $/ \mathrm{ml}$ before addition of $20 \%$ galactose to a final concentration of $2 \%$. After an additional 1 hour of incubation at $30^{\circ} \mathrm{C}$ appropriate dilutions were plated onto YPD and incubated at $30^{\circ} \mathrm{C}$ for three days. The resulting colonies were counted and replica plated onto fresh YPD plates, printed with a lawn of a MATa tester strain (R113a) and incubated overnight at $30^{\circ} \mathrm{C}$. Incubated prints were replica plated onto synthetic dextrose minimal medium (SD) and incubated overnight at $30^{\circ} \mathrm{C}$. Colonies derived from cells that had successfully converted to MAT $\alpha$ by repair of the $\mathrm{HO}$ endonuclease induced DSB at the MAT locus mated with the MATa tester lawn cells to form prototrophic diploids that showed up as patches of growing cells on SD replicas. Patches 
that overlapped with colonies on the original YPD plates were counted as recombinants. MTI frequencies were determined by dividing the number of recombinants by the total number of colonies on the original YPD plates. Mean MTI frequencies, $95 \%$ confidence intervals and t-test values were calculated with Prism. A select number of putative diploids from each plating were replica plated to sporulation medium and tetrad formation was scored by microscopic examination, demonstrating the presence of functional MATa and MAT $\alpha$ alleles, and indicating successful mating type interconversion (i.e. MATa to MAT $\alpha$ ) in the cells generating the original colonies.

\section{DSB-stimulated ectopic gene conversion (EGC) frequency determination}

The frequency of repair of a $\mathrm{HO}$ endonuclease catalyzed DSB at the HIS3 locus on chromosome XV (his3- $\left.\triangle 3^{\prime}-H O c s\right)$ by conservative homologous recombination with a his $3-\Delta M s c l$ allele proximal to the LEU2 locus on chromosome III was determined as described previously [29]. A minimum of 10 one milliliter YPGL cultures were inoculated with single colonies of selected strains and grown at $30^{\circ} \mathrm{C}$ overnight to a density of 1 $-2 \times 10^{7}$ cells $/ \mathrm{ml}$ before addition of $20 \%$ galactose to a final concentration of $2 \%$ followed by an additional four hour incubation at $30^{\circ} \mathrm{C}$. Dilutions were plated onto YPD medium to assess viability and onto synthetic complete medium lacking histidine (SC-His) to select for recombinants. Plates were incubated at $30^{\circ} \mathrm{C}$ for three days and the number of colonies counted. Frequencies of EGC were determined by dividing the number of $\mathrm{His}^{+}$recombinants by the number of viable cells plated. Mean EGC frequencies, 95\% confidence intervals and t-test values were calculated with Prism. Gene conversion events at the HIS3 locus in select $\mathrm{His}^{+}$recombinants were verified by Southern blot analysis.

\section{Assaying recombination between non-tandem direct repeats} We determined the frequency of repair of a $\mathrm{HO}$-catalyzed DSB by non-conservative HR between 3 '- and $5^{\prime}$-truncated copies of the HIS3 coding sequence flanking the URA3-marked plasmid YIp5 inserted into the HIS3 locus on chromosome XV [95]. Single colonies of select strains grown on SC medium lacking uracil (SC-Ura) were used to inoculate a minimum of 10 one milliliter YPGL cultures and grown overnight to a density of 1 $2 \times 10^{7}$ cells $/ \mathrm{ml}$. Addition of $20 \%$ galactose to a final concentration of $2 \%$ with additional four hour incubation at $30^{\circ} \mathrm{C}$ facilitated expression of $\mathrm{HO}$ endonuclease from trp1::GAL-HOkanMX and DSB formation at the HO cut site immediately distal to the $3^{\prime}$ truncated copy of his3 (Figure 4A). Dilutions were plated onto YPD to determine viability and onto SC-His to select for recombinants. Colonies were counted after incubation at $30^{\circ} \mathrm{C}$ for three days. Frequencies of DSB-stimulated direct repeat recombination (DSB-DRR) were determined by dividing the number of $\mathrm{His}^{+}$recombinant colonies by the number of viable cells plated. Mean DRR frequencies, $95 \%$ confidence intervals and t-test values were calculated using Prism. Select $\mathrm{His}^{+}$recombinants were scored for deletion events by replica plating to SC-Ura and/or Southern blot analysis.

We used a previously described assay [95] to determine rates of spontaneous DRR (S-DRR). We used strains with the identical $3^{\prime}$ - and 5'-truncated his3 repeats flanking the URA3marked plasmid YIp5 inserted into the HIS3 locus used above, but lacking an $\mathrm{HO}$ cut site adjacent to the $3^{\prime}$-truncated repeat (Figure S3A). Single colonies of select strains grown on SC-Ura were used to inoculate a minimum of 10 one milliliter YPD cultures and grown overnight to saturation at $30^{\circ} \mathrm{C}$ before dilutions were plated onto YPD to assess viability and SC-His to select for recombinants. Colonies were counted after incubation at $30^{\circ} \mathrm{C}$ for three days and rates of DRR determined by the method of the median [96] as described previously [97].

\section{Quantitating protein interaction by yeast two-hybrid analysis} Interaction between wild-type or mutant HsRAD52 proteins were quantitated as previously described [29]. The plasmids pGBT9 and pGAD424 and their derivatives (Table S2) were transformed into the yeast strain Y187 (Clontech, Mountain View, CA, USA) and single transformant colonies used to inoculate a minimum of 10 five milliliter culture of SC lacking leucine and tryptophan and grown to saturation at $30^{\circ} \mathrm{C}$. A standard protocol was used to prepare cell extracts and determine specific activities of $\beta$-galactosidase in Miller units. Mean specific activities, $95 \%$ confidence intervals and T-test values were calculated with Prism.

\section{Examining multimerization of HsRAD52 by gel filtration anal- ysis}

Plasmids were constructed using the vector pET28b (Novagen) for expression in bacterial cells of HsRAD521-212 (pLAY855), HsRad52-G59R $_{(1-212)}$ (pLAY971) or HsRAD52-S346X (pLAY970). Plasmids were transformed into Artic Express PR competent $E$. coli cells (Agilent, Santa Clara, CA, USA) with selection on LB medium containing kanamycin at a final concentration of 50 $\mu \mathrm{g} / \mathrm{mL}$. Transformants were used to inoculate liquid LBkanamycin cultures and grown to the appropriate density $\left(O D_{600}=0.7\right)$ before the induction of protein expression by addition of IPTG to a final concentration of $0.4 \mathrm{mM}$. Induced cells were grown at $15^{\circ} \mathrm{C}$ for 20 hours. Cell pellets were harvested by centrifugation, weighed and resuspended in $5 \mathrm{~mL}$ of $20 \mathrm{mM}$ Tris $\mathrm{HCl}, 300 \mathrm{mM} \mathrm{NaCl}, 20 \mathrm{mM}$ imidazole and $2 \mathrm{mM} \beta$ mercaptoethanol (BME), pH 8.0 buffer per gram of cells. Cells were lysed by sonication, cleared by centrifugation for $30 \mathrm{~min}$ at $30,000 \times \mathrm{g}$ and supernatants loaded onto Ni-NTA affinity columns (HisTrap FF; GE Health Sciences, Westborough, MA, USA). Protein bound to the column was eluted with a linear imidazole gradient $(700 \mathrm{mM})$. Eluted protein was either dialyzed, or fast desalted using a Hi Prep 26/10 Desalting column (GE Health Services), into $20 \mathrm{mM}$ Tris $\mathrm{HCl}, 50 \mathrm{mM} \mathrm{NaCl}, 5 \%$ glycerol, $2 \mathrm{mM}$ BME, $\mathrm{pH}$ 8.0. Soluble protein was then applied to a HiPrep Heparin FF 16/10 column (GE Health Sciences) and eluted with a $\mathrm{NaCl}$ gradient $(700 \mathrm{mM})$. Fractions containing wild-type or mutant HsRAD52 were run through a size exclusion column (Superdex 200; GE Health Sciences) preequilibrated in $10 \mathrm{mM}$ Tris $\mathrm{HCl}, 300 \mathrm{mM} \mathrm{NaCl}, 2 \mathrm{mM} \mathrm{BME}, \mathrm{pH}$ 8.0 at $0.5 \mathrm{~mL} / \mathrm{min}$ flow rate.

Determining levels of interaction of ScRad52 and HsRAD52 with donor and recipient loci during $\mathrm{MTI}$

Association of C-terminally FLAG-tagged ScRad52, or wild-type or mutant HsRAD52 with the donor HML, recipient MAT and control SAM1 loci during MTI were examined using chromatin immunoprecipitation (ChIP) as described previously [29]. Selected strains were grown in YPGL cultures and aliquots collected before and at various times after addition of galactose to a final concentration of $2 \%$. Cells were collected and extracts prepared before chromatin fragments associated with ScRad52-FLAG or HsRAD52-FLAG were immunoprecipitated, 
bound to protein $A / G$ beads, and DNA purified from eluted chromatin fragments. The amounts of specific DNA sequences adjacent to the MATa, HML and SAM1 loci among the immunoprecipitated DNA were determined by quantitative polymerase chain reaction (qPCR) using specific primer sets (Table S3). The MATa primer set amplifies a 110 bp sequence 500 bp upstream of the HO cut site in MATa on chromosome III. The HML primer set amplifies a 187 bp sequence 67 bp downstream from the $\mathrm{HO}$ cut site sequence in $\mathrm{HML}$ on chromosome III. The SAM1 primer set amplifies a $105 \mathrm{bp}$ sequence $187 \mathrm{bp}$ upstream from the SAM1 coding sequence on chromosome XII. Each qPCR determination was performed in triplicate on a BioRad CFX96 Touch Real-Time PCR Detection System (BioRad, Hercules, CA, USA) using the following cycling conditions: Step $1,95^{\circ} \mathrm{C}, 10$ minutes; Step $2,95^{\circ} \mathrm{C}, 30$ seconds; Step $3,56^{\circ} \mathrm{C}, 30$ seconds; Step 4, $72^{\circ} \mathrm{C}, 30$ seconds; Step 5, Detection; return to step 2 and repeat 39 times; Step 6, melt curve analysis from $65^{\circ} \mathrm{C}$ to $95^{\circ} \mathrm{C}$. Data were analyzed using the Bio-Rad CFX Manager 3.1. Cycle quantification $(\mathrm{Cq})$ values were reported in triplicate and outliers, defined as those outside a range of 0.5 cycles were excluded from calculations. No template controls yielded $\mathrm{Cq}$ values outside the acceptable range (>35). Association of ScRad52-FLAG, or wild-type and mutant HsRAD52-FLAG with the donor and recipient sequences was recorded as fold enrichment determined by degree of occupancy at the $H M L$ and MATa loci relative to occupancy at the SAM1 reference locus and relative to degree of occupancy before addition of galactose to the cultures $(t=0)$, and calculated by the Livak method [98]. Mean fold changes in degree of occupancy and standard deviations were calculated from at least 10 technical replicates drawn from at least three independent biological replicates. T-test values were calculated with Prism.

\section{Determining levels of repair synthesis during MTI}

Repair of the DSB at MATa using sequences from HML as template during MTI was followed over time using semiquantitative PCR with DNA sequences recovered from the same chromatin fragments prepared for ChIP analysis, but collected prior to the immunoprecipitation step. The amount of extension by repair synthesis of MAT sequences cleaved by HO-endonuclease using the intact $H M L$ locus as template was determined by PCR using the Slext-f and Slext-r primers (Table S3) as described previously [54]. These primers were chosen as they are complementary to genomic sequences unique to MAT and HML and lay as close as possible to the sequences required for $\mathrm{MTI}$. This strategy results in an amplicon that is $500 \mathrm{bp}$ in length and too long for quantitation using qPCR. The following cycling conditions were used: Step $1,95^{\circ} \mathrm{C}, 2$ minutes; Step $2,95^{\circ} \mathrm{C}, 20$ seconds; Step $3,64^{\circ} \mathrm{C}, 10$ seconds; Step $4,70^{\circ} \mathrm{C}, 10$ seconds; Step 5 , return to step 2 and repeat 34 times; Step $6,70^{\circ} \mathrm{C}, 5$ minutes. The same primers for amplifying sequences adjacent to SAM1 described above were used to generate signals for normalization using the following cycling conditions: Step $1,95^{\circ} \mathrm{C}, 10$ minutes; Step $2,95^{\circ} \mathrm{C}, 30$ seconds; Step $3,56^{\circ} \mathrm{C}, 30$ seconds; Step $4,72^{\circ} \mathrm{C}, 30$ seconds;

\section{REFERENCES}

1. Frankenberg-Schwager M, Frankenberg D (1990). DNA doublestrand breaks: their repair and relationship to cell killing in yeast. Int J Radiat Biol 58(4): 569-575. doi: 10.1080/09553009014551931
Step 5, return to step 2 and repeat 29 times; Step 6, $72^{\circ} \mathrm{C}, 5$ minutes. PCR products were separated by electrophoresis on $1.5 \%$ agarose gels, stained with ethidium bromide $(10 \mu \mathrm{g} / \mathrm{ml})$, and quantitated using ImageJ (imagej.net). Fold changes in normalized levels of repair synthesis were reported as fold differences from levels observed before addition of galactose to the growth medium $(t=0)$. Mean fold changes and standard errors were calculated from at least three independent biological replicates using Prism.

\section{ACKNOWLEDGMENTS}

This work was supported by a H. N. and Frances C. Berger Foundation Fellowship to A. D. C., funds from the Eugene and Ruth Roberts Summer Student Academy to S. O., funds from the City of Hope - Duarte High School NIH Science Education Partnership Award Program to O. L., I. Y. L. and R. R., funds from the Molecular Biology Program of Pomona College to E. W. W., J. X. and M. C. N., NIH grant R50CA211280 to A. W. A., NIH grant 1PA20CA242620-01 to J. J. P. P., the Morris and Horowitz Families Professorship to S. L. N., the Beckman Research Institute of City of Hope, and the Jefferson College of Health Professions of Thomas Jefferson University. We thank M. Boldin, R.-J. Lin, L. Malkas, J. Stark, and R. Rothstein for helpful discussions. We thank R. Rothstein for the gift of the R113a matingtype tester strain. We thank W. Tsark for critical assistance with setting up the X-ray irradiator. We also thank the anonymous reviewers whose comments allowed us to substantially improve the manuscript.

\section{SUPPLEMENTAL MATERIAL}

All supplemental data for this article are available online at www.microbialcell.com.

\section{CONFLICT OF INTEREST}

The authors declare that there are no conflicts of interest.

\section{COPYRIGHT}

(C) 2020 Clear et al. This is an open-access article released under the terms of the Creative Commons Attribution (CC BY) license, which allows the unrestricted use, distribution, and reproduction in any medium, provided the original author and source are acknowledged.

Please cite this article as: Alissa D. Clear, Glenn M. Manthey, Olivia Lewis, Isabelle Y. Lopez, Rossana Rico, Shannon Owens, M. Cristina Negritto, Elise W. Wolf, Jason Xu, Nikola Kenjić, J. Jefferson P. Perry, Aaron W. Adamson, Susan L. Neuhausen, Adam M. Bailis (2020). Variants of the human RAD52 gene confer defects in ionizing radiation resistance and homologous recombination repair in budding yeast. Microbial Cell 7(10): 270-285. doi: 10.15698/mic2020.10.732

2. Khanna KK, Jackson SP (2001). DNA double-strand breaks: signaling, repair and the cancer connection. Nat Genet 27(3): 247-254. doi: $10.1038 / 85798$ 
3. Pastink A, Eeken JC, Lohman PH (2001). Genomic integrity and the repair of double-strand DNA breaks. Mutat Res 480-481:37-50. doi: 10.1016/s0027-5107(01)00167-1

4. Santivasi $W L$, Xia $F$ (2014). lonizing radiation-induced DNA damage, response, and repair. Antioxid Redox Signal 21(2): 251-259. doi: 10.1089/ars.2013.5668

5. Essers J, Hendriks RW, Swagemakers SM, Troelstra C, de Wit J, Bootsma D, Hoeijmakers JH, Kanaar R (1997). Disruption of mouse $R A D 54$ reduces ionizing radiation resistance and homologous recombination. Cell 89(2): 195-204. doi: 10.1016/s00928674(00)80199-3

6. Hama-Inaba H, Saeki T (1975). Evidence that a recombinationless strain, rad 51, of Saccharomyces cerevisiae lacks the budding cell resistance to gamma-rays. J Radiat Res 16(1): 37-44. doi: 10.1269/jrr.16.37

7. Mondello C, Guasconi V, Giulotto E, Nuzzo F (2002). Gamma-ray and hydrogen peroxide induction of gene amplification in hamster cells deficient in DNA double strand break repair. DNA Repair 1(6): 483-493. doi: 10.1016/s1568-7864(02)00035-6

8. Pluth JM, Fried LM, Kirchgessner CU (2001). Severe combined immunodeficient cells expressing mutant hRAD54 exhibit a marked DNA double-strand break repair and error-prone chromosome repair defect. Cancer Res 61(6): 2649-2655. PMID: 11289143

9. Resnick MA, Martin P (1976). The repair of double-strand breaks in the nuclear DNA of Saccharomyces cerevisiae and its genetic control. Mol Gen Genet 143(2): 119-129. doi: 10.1007/bf00266917

10. Sargentini NJ, Smith KC (1986). Characterization and quantitation of DNA strand breaks requiring recA-dependent repair in X-irradiated Escherichia coli. Radiat Res 105(2): 180-186. doi: 10.2307/3576543

11. Scully R, Ganesan S, Vlasakova K, Chen J, Socolovsky M, Livingston DM (1999). Genetic analysis of BRCA1 function in a defined tumor cell line. Mol Cell 4(6): 1093-1099. doi: 10.1016/s1097-2765(00)80238-5

12. Clikeman JA, Khalsa GJ, Barton SL, Nickoloff JA (2001). Homologous recombinational repair of double-strand breaks in yeast is enhanced by MAT heterozygosity through yKU-dependent and independent mechanisms. Genetics 157(2): 579-589. PMID: 11156980.

13. Iliakis G, Mladenov E, Mladenova V (2019). Necessities in the Processing of DNA Double Strand Breaks and Their Effects on Genomic Instability and Cancer. Cancers 11(11): 1671. doi: 10.3390/cancers11111671

14. Lin Y, Lukacsovich T, Waldman AS (1999). Multiple pathways for repair of DNA double-strand breaks in mammalian chromosomes. Mol Cell Biol 19(12): 8353-8360. doi: 10.1128/mcb.19.12.8353

15. Nagasawa H, Brogan JR, Peng Y, Little JB, Bedford JS (2010). Some unsolved problems and unresolved issues in radiation cytogenetics: a review and new data on roles of homologous recombination and nonhomologous end joining. Mutat Res 701(1): 12-22. doi: 10.1016/j.mrgentox.2010.03.003

16. Schipler A, lliakis G (2013). DNA double-strand-break complexity levels and their possible contributions to the probability for errorprone processing and repair pathway choice. Nucleic Acids Res 41(16): 7589-7605. doi: 10.1093/nar/gkt556

17. Chistiakov DA, Voronova NV, Chistiakov PA (2008). Genetic variations in DNA repair genes, radiosensitivity to cancer and susceptibility to acute tissue reactions in radiotherapy-treated cancer patients. Acta Oncologica 47(5): 809-824. doi: 10.1080/02841860801885969

18. Hromas R, Kim HS, Sidhu G, Williamson E, Jaiswal A, Totterdale TA, Nole J, Lee SH, Nickoloff JA, Kong KY (2017). The endonuclease EEPD1 mediates synthetic lethality in RAD52-depleted BRCA1 mutant breast cancer cells. Breast Cancer Res 19(1): 122. doi: 10.1186/s13058-0170912-8

19. Lok BH, Carley AC, Tchang B, Powell SN (2013). RAD52 inactivation is synthetically lethal with deficiencies in BRCA1 and PALB2 in addition to BRCA2 through RAD51-mediated homologous recombination. Oncogene 32(30): 3552-3558. doi: 10.1038/onc.2012.391

20. Feng Z, Scott SP, Bussen W, Sharma GG, Guo G, Pandita TK, Powell SN (2011). Rad52 inactivation is synthetically lethal with BRCA2 deficiency. Proc Natl Acad Sci U S A 108(2): 686-691. doi: 10.1073/pnas.1010959107

21. Lok BH, Powell SN (2012). Molecular pathways: understanding the role of Rad52 in homologous recombination for therapeutic advancement. Clin Cancer Res 18(23): 6400-6406. doi: 10.1158/1078 0432.CCR-11-3150

22. van Veelen LR, Essers J, van de Rakt MW, Odijk $H$, Pastink $A$, Zdzienicka MZ, Paulusma CC, Kanaar R (2005). Ionizing radiationinduced foci formation of mammalian Rad51 and Rad54 depends on the Rad51 paralogs, but not on Rad52. MutatRes 574(1-2): 34-49. doi: 10.1016/j.mrfmmm.2005.01.020

23. Chandramouly G, McDevitt S, Sullivan K, Kent T, Luz A, Glickman JF, Andrake M, Skorski T, Pomerantz RT (2015). Small-Molecule Disruption of RAD52 Rings as a Mechanism for Precision Medicine in BRCA-Deficient Cancers. Chem Biol 22(11): 1491-1504. doi: 10.1016/j.chembiol.2015.10.003

24. Cramer-Morales K, Nieborowska-Skorska M, Scheibner K, Padget M, Irvine DA, Sliwinski T, Haas K, Lee J, Geng H, Roy D, Slupianek A Rassool FV, Wasik MA, Childers W, Copland M, Muschen M, Civin Cl, Skorski T (2013). Personalized synthetic lethality induced by targeting RAD52 in leukemias identified by gene mutation and expression profile. Blood 122(7): 1293-1304. doi: 10.1182/blood-2013-05-501072

25. Hengel SR, Malacaria E, Folly da Silva Constantino L, Bain FE, Diaz A, Koch BG, Yu L, Wu M, Pichierri P, Spies MA, Spies M (2016). Smallmolecule inhibitors identify the RAD52-sSDNA interaction as critical for recovery from replication stress and for survival of BRCA2 deficient cells. elife 5: e14740. doi: 10.7554/eLife.14740

26. Huang F, Goyal N, Sullivan K, Hanamshet K, Patel M, Mazina OM Wang CX, An WF, Spoonamore J, Metkar S, Emmitte KA, Cocklin S, Skorski T, Mazin AV (2016). Targeting BRCA1- and BRCA2-deficient cells with RAD52 small molecule inhibitors. Nucleic Acids Res 44(9): 4189-4199. doi: 10.1093/nar/gkw087

27. Sullivan K, Cramer-Morales K, McElroy DL, Ostrov DA, Haas K, Childers W, Hromas R, Skorski $T$ (2016). Identification of a Small Molecule Inhibitor of RAD52 by Structure-Based Selection. PLoS One 11(1): e0147230. doi: 10.1371/journal.pone.0147230

28. Sullivan-Reed K, Bolton-Gillespie E, Dasgupta $Y$, Langer S, Siciliano M, Nieborowska-Skorska M, Hanamshet K, Belyaeva EA, Bernhardy AJ, Lee J, Moore $\mathrm{M}$, Zhao $\mathrm{H}$, Valent $\mathrm{P}$, Matlawska-Wasowska $\mathrm{K}$, Muschen M, Bhatia S, Bhatia R, Johnson N, Wasik MA, Mazin AV, Skorski T (2018). Simultaneous Targeting of PARP1 and RAD52 Triggers Dual Synthetic Lethality in BRCA-Deficient Tumor Cells. Cell Rep 23(11): 3127-3136. doi: 10.1016/j.celrep.2018.05.034

29. Manthey GM, Clear AD, Liddell LC, Negritto MC, Bailis AM (2017). Homologous recombination in budding yeast expressing the human RAD52 gene reveals a Rad51-independent mechanism of conservative double-strand break repair Nucleic Acids Res 45(4): 1879-1888. doi: 10.1093/nar/gkw1228

30. Adamson AW, Ding YC, Mendez-Dorantes C, Bailis AM, Stark JM, Neuhausen SL (2020). The RAD52-S346X variant reduces risk of developing breast cancer in carriers of pathogenic germline $B R C A 2$ mutations. Mol Oncol 14(6):1124-1133. doi: 10.1002/18780261.12665 
31. Miki Y, Swensen J, Shattuck-Eidens D, Futreal PA, Harshman K, Tavtigian S, Liu Q, Cochran C, Bennett LM, Ding W, et al. (1994). A strong candidate for the breast and ovarian cancer susceptibility gene BRCA1. Science 266(5182): 66-71. doi: 10.1126/science.7545954

32. Moynahan ME, Chiu JW, Koller BH, Jasin M (1999). Brca1 controls homology-directed DNA repair. Mol Cell 4(4): 511-518. doi: 10.1016/s1097-2765(00)80202-6

33. Moynahan ME, Pierce AJ, Jasin M (2001). BRCA2 is required for homology-directed repair of chromosomal breaks. Mol Cell 7(2): 263272. doi: 10.1016/s1097-2765(01)00174-5

34. Wooster R, Neuhausen SL, Mangion J, Quirk Y, Ford D, Collins N, Nguyen K, Seal S, Tran T, Averill D, et al. (1994). Localization of a breast cancer susceptibility gene, BRCA2, to chromosome 13q12-13. Science 265(5181): 2088-2090. doi: 10.1126/science.8091231

35. Game JC, Mortimer RK (1974). A genetic study of x-ray sensitive mutants in yeast. Mutat Res 24(3): 281-292. doi: 10.1016/00275107(74)90176-6

36. Ho KS (1975). Induction of DNA double-strand breaks by X-rays in a radiosensitive strain of the yeast Saccharomyces cerevisiae. Mutat Res 30(3): 327-334. doi: 10.1016/0027-5107(75)90003-2

37. McGill C, Shafer B, Strathern J (1989). Coconversion of flanking sequences with homothallic switching. Cell 57(3): 459-467. doi: 10.1016/0092-8674(89)90921-5

38. Strathern JN, Klar AJ, Hicks JB, Abraham JA, Ivy JM, Nasmyth KA, McGill C (1982). Homothallic switching of yeast mating type cassettes is initiated by a double-stranded cut in the MAT locus. Cell 31(1): 183192. doi: 10.1016/0092-8674(82)90418-4

39. White $\mathrm{Cl}$, Haber JE (1990). Intermediates of recombination during mating type switching in Saccharomyces cerevisiae. EMBO J 9(3): 663673. doi: 10.1002/j.1460-2075.1990.tb08158.x

40. Malone RE, Esposito RE (1980). The RAD52 gene is required for homothallic interconversion of mating types and spontaneous mitotic recombination in yeast. Proc Natl Acad Sci U S A 77(1): 503-507. doi: 10.1073/pnas.77.1.503

41. Fishman-Lobell J, Rudin N, Haber JE (1992). Two alternative pathways of double-strand break repair that are kinetically separable and independently modulated. Mol Cell Biol 12(3): 1292-1303. doi: 10.1128/mcb.12.3.1292

42. Stark JM, Pierce AJ, Oh J, Pastink A, Jasin M (2004). Genetic steps of mammalian homologous repair with distinct mutagenic consequences. Mol Cell Biol 24(21): 9305-9316. doi: 10.1128/MCB.24.21.9305-9316.2004

43. Ivanov EL, Sugawara N, Fishman-Lobell J, Haber JE (1996). Genetic requirements for the single-strand annealing pathway of doublestrand break repair in Saccharomyces cerevisiae. Genetics 142(3): 693704. PMID: 8849880.

44. Tsukamoto M, Yamashita K, Miyazaki T, Shinohara M, Shinohara A (2003). The N-terminal DNA-binding domain of Rad52 promotes RAD51-independent recombination in Saccharomyces cerevisiae. Genetics 165(4): 1703-1715. PMID: 14704160.

45. Manthey GM, Bailis AM (2010). Rad51 inhibits translocation formation by non-conservative homologous recombination in Saccharomyces cerevisiae. PLoS ONE 5(7): e11889. doi: 10.1371/journal.pone.0011889

46. Mortensen UH, Erdeniz N, Feng Q, Rothstein R (2002). A molecular genetic dissection of the evolutionarily conserved $\mathrm{N}$ terminus of yeast Rad52. Genetics 161(2): 549-562. PMID: 12072453.

47. Shen Z, Cloud KG, Chen DJ, Park MS (1996). Specific interactions between the human RAD51 and RAD52 proteins. J Biol Chem 271(1): 148-152. doi: 10.1074/jbc.271.1.148
48. Shen Z, Peterson SR, Comeaux JC, Zastrow D, Moyzis RK, Bradbury EM, Chen DJ (1996). Self-association of human RAD52 protein. Mutat Res 364(2): 81-89. doi: 10.1016/0921-8777(96)00025-0

49. Van Dyck E, Hajibagheri NM, Stasiak A, West SC (1998). Visualisation of human Rad52 protein and its complexes with hRad51 and DNA. J Mol Biol 284(4): 1027-1038. doi: 10.1006/jmbi.1998.2203

50. Van Dyck E, Stasiak AZ, Stasiak A, West SC (1999). Binding of double-strand breaks in DNA by human Rad52 protein. Nature 398(6729): 728-731. doi: 10.1038/19560

51. Kagawa W, Kurumizaka H, Ishitani R, Fukai S, Nureki O, Shibata T, Yokoyama $S$ (2002). Crystal structure of the homologous-pairing domain from the human Rad52 recombinase in the undecameric form. Mol Cell 10(2): 359-371. doi: 10.1016/s-1097-2765(02)00587-7

52. Singleton MR, Wentzell LM, Liu Y, West SC, Wigley DB (2002). Structure of the single-strand annealing domain of human RAD52 protein. Proc Natl Acad Sci U S A 99(21): 13492-13497. doi: $10.1073 /$ pnas. 212449899

53. Wolner B, van Komen S, Sung P, Peterson CL (2003). Recruitment of the recombinational repair machinery to a DNA double-strand break in yeast. Mol Cell 12(1): 221-232. doi: 10.1016/s10972765(03)00242-9

54. Sugawara N, Wang X, Haber JE (2003). In vivo roles of Rad52, Rad54, and Rad55 proteins in Rad51-mediated recombination. Mol Cell 12(1): 209-219. doi: 10.1016/s1097-2765(03)00269-7

55. Iyer LM, Koonin EV, Aravind L (2002). Classification and evolutionary history of the single-strand annealing proteins, RecT, Redbeta, ERF and RAD52. BMC Genomics 3:8. doi: 10.1186/14712164-3-8

56. Kagawa W, Kagawa A, Saito K, Ikawa S, Shibata T, Kurumizaka H, Yokoyama $S$ (2008). Identification of a second DNA binding site in the human Rad52 protein. J Biol Chem 283(35): 24264-24273. doi: 10.1074/jbc.M802204200

57. Ploquin $M$, Bransi $A$, Paquet ER, Stasiak $A Z$, Stasiak $A, Y u X$, Cieslinska AM, Egelman EH, Moineau S, Masson JY (2008). Functiona and structural basis for a bacteriophage homolog of human RAD52. Curr Biol 18(15): 1142-1146. doi: 10.1016/j.cub.2008.06.071

58. Mortensen UH, Bendixen C, Sunjevaric I, Rothstein R (1996). DNA strand annealing is promoted by the yeast Rad52 protein. Proc Natl Acad Sci U S A 93(20): 10729-10734. doi: 10.1073/pnas.93.20.10729

59. Reddy G, Golub El, Radding CM (1997). Human Rad52 protein promotes single-strand DNA annealing followed by branch migration. Mutat Res 377(1): 53-59. doi: 10.1016/s0027-5107(97)00057-2

60. Shinohara A, Shinohara M, Ohta T, Matsuda S, Ogawa T (1998). Rad52 forms ring structures and co-operates with RPA in single-strand DNA annealing. Genes Cells 3(3): 145-156. doi: 10.1046/j.13652442.1998.00176.x

61. Sugiyama T, New JH, Kowalczykowski SC (1998). DNA annealing by RAD52 protein is stimulated by specific interaction with the complex of replication protein A and single-stranded DNA. Proc Natl Acad Sci U S A 95(11): 6049-6054. doi: 10.1073/pnas.95.11.6049

62. Paques F, Haber JE (1999). Multiple pathways of recombination induced by double-strand breaks in Saccharomyces cerevisiae. Microbiol Mol Biol Rev 63(2): 349-404. doi: 10.1128/mmbr.63.2.349404.1999

63. Prakash S, Prakash L, Burke W, Montelone BA (1980). Effects of the RAD52 Gene on Recombination in SACCHAROMYCES CEREVISIAE. Genetics 94(1): 31-50. doi: 10.2172/6162720

64. Symington LS (2002). Role of RAD52 epistasis group genes in homologous recombination and double-strand break repair. Microbiol Mol Biol Rev 66(4): 630-670. doi: 10.1128/mmbr.66.4.630-670.2002 
65. Jensen RB, Carreira A, Kowalczykowski SC (2010). Purified human BRCA2 stimulates RAD51-mediated recombination. Nature 467(7316) 678-683. doi: 10.1038/nature09399

66. Haber JE (2012). Mating-type genes and MAT switching in Saccharomyces cerevisiae. Genetics 191(1): 33-64. doi: 10.1534/genetics.111.134577

67. Sprague GF, Blair LC, Thorner J (1983). Cell Interactions and Regulation of Cell Type in the Yeast Saccharomyces cerevisiae. Ann Rev Microbiol 37: 623-660. doi: 10.1146/annurev.mi.37.100183.003203

68. Eichmiller R, Medina-Rivera M, DeSanto R, Minca E, Kim C, Holland C, Seol JH, Schmit M, Oramus D, Smith J, Gallardo IF, Finkelstein IJ, Lee SE, Surtees JA (2018). Coordination of Rad1-Rad10 interactions with Msh2-Msh3, Saw1 and RPA is essential for functional 3' nonhomologous tail removal. Nucleic Acids Res 46(10): 5075-5096. doi: 10.1093/nar/gky254

69. Fishman-Lobell J, Haber JE (1992). Removal of nonhomologous DNA ends in double-strand break recombination: the role of the yeast ultraviolet repair gene RAD1. Science 258(5081): 480-484. doi: 10.1126/science. 1411547

70. Li F, Dong J, Pan X, Oum JH, Boeke JD, Lee SE (2008). Microarraybased genetic screen defines $S A W 1$, a gene required for Rad1/Rad10dependent processing of recombination intermediates. Mol Cell 30(3): 325-335. doi: 10.1016/j.molcel.2008.02.028

71. Lyndaker AM, Alani E (2009). A tale of tails: insights into the coordination of 3 ' end processing during homologous recombination. Bioessays 31(3): 315-321. doi: 10.1002/bies.200800195

72. Manthey GM, Naik N, Bailis AM (2009). Msh2 Blocks an Alternative Mechanism for Non-Homologous Tail Removal During Single-Strand Annealing in Saccharomyces cerevisiae. PLoS ONE 4(10): e7488. doi: 10.1371/journal.pone.0007488

73. Pannunzio NR, Manthey GM, Bailis AM (2010). RAD59 and RAD1 cooperate in translocation formation by single-strand annealing in Saccharomyces cerevisiae. Curr Genet 56(1): 87-100. doi: 10.1007/s00294-009-0282-6

74. Sugawara N, Ira G, Haber JE (2000). DNA length dependence of the single-strand annealing pathway and the role of Saccharomyces cerevisiae RAD59 in double-strand break repair. Mol Cell Biol 20(14): 5300-5309. doi: 10.1128/mcb.20.14.5300-5309.2000

75. Sugawara N, Paques F, Colaiacovo M, Haber JE (1997). Role of Saccharomyces cerevisiae Msh2 and Msh3 repair proteins in doublestrand break-induced recombination. Proc Natl Acad Sci U S A 94(17): 9214-9219. doi: 10.1073/pnas.94.17.9214

76. Thomas BJ, Rothstein R (1989). The genetic control of directrepeat recombination in Saccharomyces: the effect of rad52 and rad1 on mitotic recombination at GAL10, a transcriptionally regulated gene. Genetics 123(4): 725-738. PMID: 2693208

77. Silva MC, Morrical MD, Bryan KE, Averill AM, Dragon J, Bond JP, Morrical SW (2016). RAD51 variant proteins from human lung and kidney tumors exhibit DNA strand exchange defects. DNA Repair 42: 44-55. doi: 10.1016/j.dnarep.2016.02.008

78. Anantha RW, Simhadri S, Foo TK, Miao S, Liu J, Shen Z, Ganesan S, Xia B (2017). Functional and mutational landscapes of BRCA1 for homology-directed repair and therapy resistance. eLife 6: e21350. doi: 10.7554/eLife. 21350

79. Silva MC, Bryan KE, Morrical MD, Averill AM, Dragon J, Wiegmans AP, Morrical SW (2017). Defects in recombination activity caused by somatic and germline mutations in the multimerization/BRCA2 binding region of human RAD51 protein. DNA Repair 60: 64-76. doi: 10.1016/j.dnarep.2017.10.008
80. Koike M, Yutoku Y, Koike A (2013). The C-terminal region of Rad52 is essential for Rad52 nuclear and nucleolar localization, and accumulation at DNA damage sites immediately after irradiation. Biochem Biophys Res Commun 435(2): 260-266. doi: 10.1016/j.bbrc.2013.04.067

81. Pannunzio NR, Manthey GM, Liddell LC, Fu BX, Roberts CM, Bailis AM (2012). Rad59 regulates association of Rad52 with DNA doublestrand breaks. Microbiologyopen 1(3): 285-297. doi: 10.1002/mbo3.31

82. Brouwer I, Zhang $H$, Candelli A, Normanno D, Peterman EJ, Wuite GJ, Modesti M (2017). Human RAD52 Captures and Holds DNA Strands, Increases DNA Flexibility, and Prevents Melting of Duplex DNA: Implications for DNA Recombination. Cell Rep 18(12): 2845 2853. doi: 10.1016/j.celrep.2017.02.068

83. Navadgi VM, Dutta A, Rao BJ (2003). Human Rad52 facilitates a three-stranded pairing that follows no strand exchange: a novel pairing function of the protein. Biochem 42(51): 15237-15251. doi: 10.1021/bi0350452

84. Sotiriou SK, Kamileri I, Lugli N, Evangelou K, Da-Re C, Huber F, Padayachy L, Tardy S, Nicati NL, Barriot S, Ochs F, Lukas C, Lukas J, Gorgoulis VG, Scapozza L, Halazonetis TD (2016). Mammalian RAD52 Functions in Break-Induced Replication Repair of Collapsed DNA Replication Forks. Mol Cell 64(6): 1127-1134. doi: 10.1016/j.molcel.2016.10.038

85. Stasiak AZ, Larquet E, Stasiak A, Muller S, Engel A, Van Dyck E, West SC, Egelman EH (2000). The human Rad52 protein exists as a heptameric ring. Curr Biol 10(6): 337-340. doi: 10.1016/s09609822(00)00385-7

86. Ranatunga W, Jackson D, Lloyd JA, Forget AL, Knight KL, Borgstahl GE (2001). Human RAD52 exhibits two modes of self-association. J Biol Chem 276(19): 15876-15880. doi: 10.1074/jbc.M011747200

87. Bhowmick R, Minocherhomji S, Hickson ID (2016). RAD52 Facilitates Mitotic DNA Synthesis Following Replication Stress. Mol Cell 64(6): 1117-1126. doi: 10.1016/j.molcel.2016.10.037

88. Toma M, Sullivan-Reed K, Sliwinski T, Skorski T (2019). RAD52 as a Potential Target for Synthetic Lethality-Based Anticancer Therapies. Cancers 11(10): 1561. doi: 10.3390/cancers11101561

89. Dhawan M, Ryan CJ, Ashworth A (2016). DNA Repair Deficiency Is Common in Advanced Prostate Cancer: New Therapeutic Opportunities. Oncologist 21(8): 940-945. doi: 10.1634/theoncologist.2016-0135

90. Konstantinopoulos PA, Ceccaldi R, Shapiro GI, D'Andrea AD (2015). Homologous Recombination Deficiency: Exploiting the Fundamental Vulnerability of Ovarian Cancer. Cancer Discov 5(11): 1137-1154. doi: 10.1158/2159-8290.CD-15-0714

91. Reilly NM, Novara L, Di Nicolantonio F, Bardelli A (2019). Exploiting DNA repair defects in colorectal cancer. Mol Oncol 13(4): 681-700. doi: 10.1002/1878-0261.12467

92. Young K, Starling N, Cunningham D (2016). Targeting deficient DNA damage repair in gastric cancer. Expert Opin Pharmacother 17(13): 1757-1766. doi: 10.1080/14656566.2016.1217992

93. Sherman F, Fink GR, Hicks JB (1986). Methods in yeast genetics. Cold Spring Harbor Laboratory Press, Cold Spring Harbor, NY.

94. Sambrook JaR, D. (2001). Molecular Cloning: A Laboratory Manual, 3rd ed. Cold Spring Harbor Laboratory Press, Cold Spring Harbor, NY.

95. Maines S, Negritto MC, Wu X, Manthey GM, Bailis AM (1998) Novel mutations in the RAD3 and SSL1 genes perturb genome stability by stimulating recombination between short repeats in Saccharomyces cerevisiae. Genetics 150(3): 963-976. PMID: 9799251. 
96. Lea DE, Coulson CA (1949). The distribution of numbers of mutants in bacterial populations. J Genet 49: 264-285. doi: 10.107/BF02986080

97. Spell RM, Jinks-Robertson S (2004). Determination of mitotic recombination rates by fluctuation analysis in Saccharomyces cerevisiae. Methods Mol Biol 262: 3-12. doi: 10.1385/1-59259-761$0: 003$

98. Livak KJ, Schmittgen TD (2001). Analysis of relative gene expression data using real-time quantitative PCR and the 2(-Delta Delta C(T)) Method. Methods 25(4): 402-408. doi: 10.1006/meth.2001.1262 